\title{
METRICALLY COMPLETE REGULAR RINGS
}

BY

\author{
K. R. GOODEARL ${ }^{1}$
}

\begin{abstract}
ABSTRACr. This paper is concerned with the structure of those (von Neumann) regular rings $R$ which are complete with respect to the weakest metric derived from the pseudo-rank functions on $R$, known as the $N^{*}$-metric. It is proved that this class of regular rings includes all regular rings with bounded index of nilpotence, and all $\boldsymbol{\aleph}_{0}$-continuous regular rings. The major tool of the investigation is the partially ordered Grothendieck group $K_{0}(R)$, which is proved to be an archimedean normcomplete interpolation group. Such a group has a precise representation as affine continuous functions on a Choquet simplex, from earlier work of the author and D. E. Handelman, and additional aspects of its structure are derived here. These results are then translated into ring-theoretic results about the structure of $R$. For instance, it is proved that the simple homomorphic images of $R$ are right and left self-injective rings, and $R$ is a subdirect product of these simple self-injective rings. Also, the isomorphism classes of the finitely generated projective $R$-modules are determined by the isomorphism classes modulo the maximal two-sided ideals of $R$. As another example of the results derived, it is proved that if all simple artinian homomorphic images of $R$ are $n \times n$ matrix rings (for some fixed positive integer $n$ ), then $R$ is an $n \times n$ matrix ring.
\end{abstract}

All rings in this paper are associative with 1 , and all modules are unital right modules. For the overall theory of regular rings, we refer the reader to [2]; for the general development of $K_{0}$ of regular rings as partially ordered abelian groups, and the theory of partially ordered abelian groups via their state spaces, we refer the reader to $[2,4]$. In particular, these references should be consulted for more detail on definitions and concepts which are just sketched here.

I. $N^{*}$-completeness. Completeness of a regular ring with respect to a rank function, or with respect to a family of pseudo-rank functions, implies that the ring is right and left self-injective [2, Theorems 19.7 and 20.8], hence a considerable amount of structure theory is available for such rings [2, Chapters 9-12]. The purpose of this paper is to investigate a much broader class of regular rings, namely those which are complete with respect to the (pseudo-) metric obtained from the supremum $N^{*}$ of all pseudo-rank functions on the ring. In particular, all regular rings complete with respect to a family of pseudo-rank functions are $N^{*}$-complete, but also, as we prove later in this section, all regular rings with bounded index of nilpotence and all $\aleph_{0}$-continuous regular rings are $N^{*}$-complete.

Received by the editors January 13, 1981 and, in revised form, April 3, 1981.

1980 Mathematics Subject Classification. Primary 16A30, 16A54, 06F20.

Key words and phrases. Regular ring, $N^{*}$-complete, $K_{0}$, interpolation group.

${ }^{1}$ This research was partially supported by a grant from the National Science Foundation. 
In addition to these results, the present section introduces the definition and a few basic properties of $N^{*}$-completeness. $\S \mathrm{II}$ is devoted to proving that $K_{0}$ of any $N^{*}$-complete regular ring is an archimedean norm-complete interpolation group, while the third section develops a number of structural properties of such groups. In $\S \mathrm{IV}$, we apply these results to the structure theory of $N^{*}$-complete regular rings.

DEFINITION. Recall that a pseudo-rank function on a regular ring $R$ is a map $P: R \rightarrow[0,1]$ such that (a) $P(1)=1$; (b) $P(x y) \leqslant P(x), P(y)$ for all $x, y \in R$; (c) $P(e+f)=P(e)+P(f)$ for all orthogonal idempotents $e, f \in R$. We use $\mathbf{P}(R)$ to denote the set of all pseudo-rank functions on $R$. Considered as a subset of the linear topological space $\mathbf{R}^{R}$ (which is given the product topology), $\mathbf{P}(R)$ is a compact convex set [2, Proposition 16.17]. In fact, $\mathbf{P}(R)$ is a Choquet simplex [2, Theorem 17.5].

Definition. Let $R$ be a regular ring. For each $x \in R$, we define

$$
N^{*}(x)=\sup \{P(x) \mid P \in \mathbf{P}(R)\},
$$

with the proviso that $N^{*}(x)=0$ in case $\mathbf{P}(R)$ is empty. (This definition is formally different from the definition of $N^{*}$ in [2, p. 272], but the two definitions are equivalent, as follows from [2, Proposition 18.10].) Thus $N^{*}(x)$ is a real number, and $0 \leqslant N^{*}(x) \leqslant 1$. Whenever the ring $R$ needs to be emphasized, we shall write $N_{R}^{*}(x)$ in place of $N^{*}(x)$. In case $R$ is unit-regular, $N^{*}$ may be computed as in the following proposition. We first recall two pieces of notation.

Definition. Given modules $A$ and $B$, we write $A \lesssim B$ to mean that $A$ is isomorphic to a submodule of $B$. Given a module $A$ and a positive integer $n$, we write $n A$ to denote the direct sum of $n$ copies of $A$.

PROPOSITION 1.1. If $R$ is a nonzero unit-regular ring, then

$$
N^{*}(x)=\inf \left\{k / n \mid k, n \in \mathbf{N} \text { and } n(x R) \lesssim k R_{R}\right\}
$$

for all $x \in R$.

Proof. [2, Proposition 18.10].

LEMMA 1.2. Let $R$ be a regular ring, and let $x, y, y_{1}, \ldots, y_{n} \in R$.

(a) If $t(x R) \lesssim s_{1}\left(y_{1} R\right) \oplus \cdots \oplus s_{n}\left(y_{n} R\right)$ for some positive integers $t, s_{1}, \ldots, s_{n}$, then

$$
N^{*}(x) \leqslant\left(s_{1} / t\right) N^{*}\left(y_{1}\right)+\cdots+\left(s_{n} / t\right) N^{*}\left(y_{n}\right) .
$$

(b) If $t(x R) \cong s(y R)$ for some positive integers $s, t$, then $N^{*}(x)=(s / t) N^{*}(y)$.

(c) $N^{*}(x y) \leqslant N^{*}(x)$ and $N^{*}(x y) \leqslant N^{*}(y)$.

(d) $N^{*}(x+y) \leqslant N^{*}(x)+N^{*}(y)$.

Proof. These results are clear if $\mathbf{P}(R)$ is empty, so assume it is nonempty.

(a) For any $P \in \mathbf{P}(R)$, we have

$$
t P(x) \leqslant s_{1} P\left(y_{1}\right)+\cdots+s_{n} P\left(y_{n}\right)
$$

by [2, Proposition 16.1], whence

$$
\begin{aligned}
P(x) & \leqslant\left(s_{1} / t\right) P\left(y_{1}\right)+\cdots+\left(s_{n} / t\right) P\left(y_{n}\right) \\
& \leqslant\left(s_{1} / t\right) N^{*}\left(y_{1}\right)+\cdots+\left(s_{n} / t\right) N^{*}\left(y_{n}\right) .
\end{aligned}
$$

Consequently, $N^{*}(x) \leqslant\left(s_{1} / t\right) N^{*}\left(y_{1}\right)+\cdots+\left(s_{n} / t\right) N^{*}\left(y_{n}\right)$. 
(b) This follows directly from (a).

(c) For all $P \in \mathbf{P}(R)$, we have $P(x y) \leqslant P(x) \leqslant N^{*}(x)$, hence $N^{*}(x y) \leqslant N^{*}(x)$. Likewise, $N^{*}(x y) \leqslant N^{*}(y)$.

(d) Since $(x+y) R \leqslant x R+y R \lesssim x R \oplus y R$, we may apply (a).

Definition. Let $R$ be a regular ring. In view of Lemma 1.2, we see that the rule $\delta(x, y)=N^{*}(x-y)$ defines a pseudo-metric $\delta$ on $R$. By way of abbreviation, we shall refer to $\delta$ as the $N^{*}$-metric on $R$, even though $\delta$ is not always a metric. Note that $\delta$ is a metric if and only if $\operatorname{ker}(\operatorname{P}(R))=0$. For all $x, y, z, w \in R$, we see that

$$
\begin{gathered}
N^{*}((x+y)-(z+w))=N^{*}((x-z)+(y-w)) \leqslant N^{*}(x-z)+N^{*}(y-w), \\
N^{*}(x y-z w)=N^{*}((x-z) y+z(y-w)) \leqslant N^{*}(x-z)+N^{*}(y-w) .
\end{gathered}
$$

Thus addition and multiplication in $R$ are uniformly continuous with respect to $N^{*}$. For all $x, y \in R$, we also have

$$
\begin{gathered}
N^{*}(x) \leqslant N^{*}(x-y)+N^{*}(y), \\
N^{*}(y) \leqslant N^{*}(y-x)+N^{*}(x)=N^{*}(x-y)+N^{*}(y),
\end{gathered}
$$

whence $\left|N^{*}(x)-N^{*}(y)\right| \leqslant N^{*}(x-y)$. Thus the map $N^{*}: R \rightarrow[0,1]$ is uniformly continuous with respect to the $N^{*}$-metric.

Definition. A regular ring $R$ is called $N^{*}$-complete provided $\operatorname{ker}(\mathrm{P}(R))=0$ (so that the $N^{*}$-metric on $R$ is actually a metric) and $R$ is complete in the $N^{*}$-metric. For example, if $R$ is a simple artinian ring, then there is a unique rank function $P$ on $R$, which takes on only the values $0,1 / k, 2 / k, \ldots, 1$, for some $k \in \mathbf{N}$ [2, Corollary 16.6]. As a result, $N^{*}=P$, and $N^{*}(x) \geqslant 1 / k$ for all nonzero $x \in R$, so that the $N^{*}$-metric on $R$ is discrete. Therefore $R$ is $N^{*}$-complete. More generally, we have the following result.

THEOREM 1.3. A regular ring $R$ has bounded index of nilpotence if and only if $\operatorname{ker}(\mathbf{P}(R))=0$ and the $N^{*}$-metric on $R$ is discrete, in which case $R$ is $N^{*}$-complete.

Proof. First assume that $\operatorname{ker}(\mathbf{P}(R))=0$ and the $N^{*}$-metric on $R$ is discrete. Then there exists $n \in \mathbf{N}$ such that $N^{*}(x) \geqslant 1 / n$ for any nonzero $x \in R$, and we claim that the index of nilpotence of $R$ is at most $n$. If not, $R$ must contain a direct sum of $n+1$ nonzero pairwise isomorphic right ideals [2, Theorem 7.2]. Consequently, there is a nonzero element $y \in R$ satisfying $(n+1)(y R) \lesssim R_{R}$. But then $N^{*}(y)>0$ (because $\operatorname{ker}(\mathbf{P}(R))=0)$ and $N^{*}(y) \leqslant 1 /(n+1)$ (by Lemma 1.2), contradicting our discreteness assumption. Therefore the index of nilpotence of $R$ is at most $n$, as claimed.

Conversely, assume that $R$ has bounded index of nilpotence. We first note that any maximal two-sided ideal $M$ of $R$ is the kernel of a pseudo-rank function on $R$. Namely, $R / M$ is a simple artinian ring [2, Theorem 7.9], so there exists a unique rank function on $R / M$, which pulls back to a pseudo-rank function on $R$ with kernel $M$.

As all primitive factor rings of $R$ are artinian [2, Corollary 7.10], the intersection of the maximal two-sided ideals of $R$ is zero. Since each maximal two-sided ideal is the kernel of a pseudo-rank function, we obtain $\operatorname{ker}(\mathbf{P}(R))=0$. 
Let $n$ be the index of nilpotence of $R$. We claim that $N^{*}(x) \geqslant 1 / n$ for any nonzero element $x \in R$.

Choose a maximal two-sided ideal $M$ of $R$ such that $x \notin M$. By [2, Theorem 7.9], $R / M \cong M_{k}(D)$ for some positive integer $k \leqslant n$ and some division ring $D$. Then $R / M$ has a unique rank function $Q$, which takes on only the values $0,1 / k, 2 / k, \ldots, 1$, and $Q(x+M) \geqslant 1 / k \geqslant 1 / n$. Pulling $Q$ back to a pseudo-rank function $P$ on $R$, we obtain $P(x) \geqslant 1 / n$, and so $N^{*}(x) \geqslant 1 / n$, as claimed.

Therefore the $N^{*}$-metric on $R$ is discrete, and, consequently, $R$ is $N^{*}$-complete.

For a deeper class of examples, we now proceed to prove that every $\boldsymbol{\aleph}_{0}$-continuous regular ring is $N^{*}$-complete. In particular, it will follow that every regular, right and left self-injective ring is $N^{*}$-complete. Recall that a regular ring $R$ is defined to be $\boldsymbol{\aleph}_{0}$-continuous provided the lattice of principal right ideals of $R$ is an $\boldsymbol{\aleph}_{0}$-continuous geometry. Equivalently, $R$ is $\aleph_{0}$-continuous if and only if every countably generated right (left) ideal of $R$ is essential in a principal right (left) ideal [2, Corollary 14.4].

We begin with a lemma generalizing [2, Corollary $14.27(a)$ ] to finitely generated projective modules. This lemma is also implicit in [8, Proposition 2.1].

LEMMA 1.4. Let $R$ be an $\boldsymbol{\aleph}_{0}$-continuous regular ring, let $A$ and $B$ be finitely generated projective right $R$-modules, and let $A_{1} \leqslant A_{2} \leqslant \ldots$ be an ascending sequence of finitely generated submodules of $A$. If $\cup A_{n}$ is essential in $A$, and each $A_{n} \lesssim B$, then $A \lesssim B$.

Proof. Let $S$ be the maximal right $\aleph_{0}$-quotient ring of $R$ [2, pp. 177, 178], so that $S$ is an $\aleph_{0}$-continuous, regular, right and left $\boldsymbol{\aleph}_{0}$-injective overring of $R$ [2, Theorems 14.12 and 14.17]. Now $A \otimes_{R} S$ and $B \otimes_{R} S$ are finitely generated projective right $S$-modules, $A \otimes_{R} S$ has an ascending sequence of finitely generated submodules which may be labelled $A_{1} \otimes_{R} S \leqslant A_{2} \otimes_{R} S \leqslant \ldots$, the submodule $\cup\left(A_{n} \otimes_{R} S\right)$ is essential in $A \otimes_{R} S$, and each $A_{n} \otimes_{R} S \lesssim B \otimes_{R} S$. Moreover, if $A \otimes_{R} S \lesssim B \otimes_{R} S$, then [2, Proposition 14.28] shows that $A \lesssim B$.

Thus there is no loss of generality in assuming that $R$ is right and left $\boldsymbol{\aleph}_{0}$-injective. Consequently, all matrix rings over $R$ are $\aleph_{0}$-continuous [2, Proposition 14.19]. Using the standard Morita-equivalences, we may transfer our problem to the category of right modules over any matrix ring $M_{k}(R)$. By choosing $k$ large enough we may arrange for the new modules corresponding to $A$ and $B$ to be cyclic, hence isomorphic to right ideals of $M_{k}(R)$.

Therefore we may now assume, without loss of generality, that $A$ and $B$ are principal right ideals of $R$. Since $\cup A_{n}$ is essential in $A$, we see that $A$ is the supremum, in the lattice of principal right ideals of $R$, of the family $\left\{A_{n}\right\}$. Consequently, [2, Corollary 14.27] shows that $A \lesssim B$.

LEMMA 1.5. Let $R$ be an $\boldsymbol{\aleph}_{0}$-continuous regular ring, and let $x, x_{1}, x_{2}, \ldots$ be elements of $R$. If the right ideal $\sum x_{n} R$ is essential in $x R$, then $N^{*}(x) \leqslant \sum N^{*}\left(x_{n}\right)$.

Proof. Choose elements $y_{1}, y_{2}, \ldots \in R$ such that $y_{n} R=x_{1} R+\cdots+x_{n} R$ for all $n$, and note from Lemma 1.2 that $N^{*}\left(y_{n}\right) \leqslant N^{*}\left(x_{1}\right)+\cdots+N^{*}\left(x_{n}\right)$. Thus it suffices 
to show that $N^{*}(x) \leqslant \sup \left\{N^{*}\left(y_{n}\right)\right\}$. If not,

$$
N^{*}(x)>s / t>\sup \left\{N^{*}\left(y_{n}\right)\right\}
$$

for some $s, t \in \mathbf{N}$.

For each $n$, we have $P\left(y_{n}\right) \leqslant N^{*}\left(y_{n}\right)<s / t$ and so $t P\left(y_{n}\right)<s P(1)$, for all $P \in$ $\mathbf{P}(R)$. As a result, [2, Theorem 18.28] implies that $t\left(y_{n} R\right) \lesssim s R_{R}$. Inside the projective module $t(x R)$, we have finitely generated submodules $t\left(y_{1} R\right) \leqslant t\left(y_{2} R\right) \leqslant$ $\ldots$, and $\cup\left(t\left(y_{n} R\right)\right)$ is an essential submodule of $t(x R)$. Consequently, Lemma 1.4 says that $t(x R) \lesssim s R_{R}$. But then $N^{*}(x) \leqslant s / t$ (by Lemma 1.2), which is false.

Therefore $N^{*}(x) \leqslant \sup \left\{N^{*}\left(y_{n}\right)\right\}$.

The key to the upcoming completeness argument is the following lemma, which is a modification of a corresponding argument of von Neumann's [10, Lemma 17.3, p. 228]. Another completeness argument using this method occurs in [2, Lemma 21.6], and we can adapt the proof of that lemma with only minor changes.

LEMMA 1.6. If $R$ is an $\aleph_{0}$-continuous regular ring and $e$ is an idempotent in $R$, then $e R(1-e)$ is complete in the $N^{*}$-metric.

Proof. We shall need the fact that $R$ is unit-regular [2, Theorem 14.24].

Let $\left\{x_{1}, x_{2}, \ldots\right\}$ be a sequence in $e R(1-e)$ which is Cauchy in the $N^{*}$-metric. By passing to a subsequence, we may assume that $N^{*}\left(x_{i}-x_{j}\right)<1 / 2^{k+1}$ whenever $i, j \geqslant k$. Set $A_{n}=\left(1-e+x_{n}\right) R$ for all $n$, and note that $A_{n}+e R=R$. For each $k=1,2, \ldots$, there exists a principal right ideal $B_{k}$ in $R$ such that

$$
\sum_{n=k}^{\infty} A_{n} \leqslant{ }_{e} B_{k}
$$

and since $A_{k} \leqslant B_{k}$, we see that $B_{k}+e R=R$. Note that $B_{1} \geqslant B_{2} \geqslant \ldots$, and set

$$
C=\bigcap_{k=1}^{\infty} B_{k} \text {. }
$$

Inasmuch as the lattice of principal right ideals of $R$ is $\boldsymbol{\aleph}_{0}$-continuous, we obtain

$$
C+e R=\left(\bigcap_{k=1}^{\infty} B_{k}\right)+e R=\bigcap_{k=1}^{\infty}\left(B_{k}+e R\right)=R .
$$

Consequently, there exists an idempotent $f \in R$ such that $f R=e R$ and $(1-f) R \leqslant$ C.

Since $f R=e R$, we have $f=e f$ and $e=f e$, hence the element $x=e-f$ lies in $e R(1-e)$. We shall show that $x_{n} \rightarrow x$ in the $N^{*}$-metric.

For each $k=1,2, \ldots$, there exists an element $d_{k} \in R$ such that

$$
\sum_{j=k+1}^{\infty}\left(x_{j}-x_{j-1}\right) R \leqslant_{e} d_{k} R
$$

and Lemma 1.5 shows that

$$
N^{*}\left(d_{k}\right) \leqslant \sum_{j=k+1}^{\infty} N^{*}\left(x_{j}-x_{j-1}\right)<\sum_{j=k+1}^{\infty} 1 / 2^{j}=1 / 2^{k}
$$


For all $n \geqslant k$, we have

$$
\begin{aligned}
A_{n} & =\left(1-e+x_{n}\right) R=\left(1-e+x_{k}+\sum_{j=k+1}^{n}\left(x_{j}-x_{j-1}\right)\right) R \\
& \leqslant\left(1-e+x_{k}\right) R+\sum_{j=k+1}^{n}\left(x_{j}-x_{j-1}\right) R \leqslant A_{k}+d_{k} R .
\end{aligned}
$$

Consequently, $\sum_{n=k}^{\infty} A_{n} \leqslant A_{k}+d_{k} R$, whence $B_{k} \leqslant A_{k}+d_{k} R$.

Each $B_{k}=A_{k} \oplus u_{k} R$ for some $u_{k} \in R$. Then

$$
A_{k} \oplus u_{k} R=B_{k} \leqslant A_{k}+d_{k} R \lesssim A_{k} \oplus d_{k} R
$$

and so $u_{k} R \lesssim d_{k} R$ (because $R$ is unit-regular). As a result, $N^{*}\left(u_{k}\right) \leqslant N^{*}\left(d_{k}\right)<1 / 2^{k}$ (Lemma 1.2).

The idempotent $1-f$ lies in the right ideal

$$
C \leqslant B_{k}=A_{k}+u_{k} R=\left(1-e+x_{k}\right) R+u_{k} R,
$$

hence $1-f=\left(1-e+x_{k}\right) r+u_{k} s$ for some $r, s \in R$. Since $x_{k} \in e R(1-e)$, we see that $1-e+x_{k}$ is idempotent, whence

$$
\begin{aligned}
\left(1-e+x_{k}\right)(1-f) & =\left(1-e+x_{k}\right) r+\left(1-e+x_{k}\right) u_{k} s \\
& =1-f+\left(x_{k}-e\right) u_{k} s .
\end{aligned}
$$

In addition, since $f R=e R$, we have $R(1-f)=R(1-e)$, and so $1-e+x_{k}$ lies in $R(1-f)$. Thus

$$
1-e+x_{k}=\left(1-e+x_{k}\right)(1-f)=1-f+\left(x_{k}-e\right) u_{k} s,
$$

and consequently

$$
x_{k}-x=x_{k}-e+f=\left(1-e+x_{k}\right)-(1-f)=\left(x_{k}-e\right) u_{k} s,
$$

hence $N^{*}\left(x_{k}-x\right) \leqslant N^{*}\left(u_{k}\right)<1 / 2^{k}$.

Therefore $x_{k} \rightarrow x$ in the $N^{*}$-metric.

We shall apply Lemma 1.6 in a situation where $R$ is a matrix ring and $e$ is a corner idempotent. For this purpose, and for later use, we need the following information concerning $N^{*}$ in matrix rings.

LEMMA 1.7. Let $R$ be a regular ring, let $n \in \mathrm{N}$, and set $T=M_{n}(R)$. Let $\varphi: R \rightarrow T$ be the natural map, and let $\left\{e_{i j} \mid i, j=1, \ldots, n\right\}$ be the standard matrix units in $T$.

(a) $N_{T}^{*} \varphi=N_{R}^{*}$.

(b) $N_{T}^{*}\left(\varphi(x) e_{i j}\right)=N_{R}^{*}(x) / n$ for all $x \in R$ and all $i, j$.

(c) $N_{R}^{*}\left(y_{i j}\right) \leqslant n N_{T}^{*}(y)$ for all $y \in T$ and all $i, j$.

(d) $N_{T}^{*}(y) \leqslant \sum_{i, j=1}^{n} N_{R}^{*}\left(y_{i j}\right) / n$ for all $y \in T$.

Proof. (a) According to [2, Corollary 16.10], the rule $P \mapsto P \varphi$ defines a bijection of $\mathbf{P}(T)$ onto $\mathbf{P}(R)$, hence

$$
N_{R}^{*}(x)=\sup \{P \varphi(x) \mid P \in \mathbf{P}(T)\}=N_{T}^{*} \varphi(x)
$$

for any $x \in R$. 
(b) For each $k=1, \ldots, n$, we note that left multiplication by $e_{k i}$ defines an isomorphism of $\varphi(x) e_{i j} T$ onto $\varphi(x) e_{k k} T$. Consequently,

$$
\varphi(x) T=\bigoplus_{k=1}^{n} \varphi(x) e_{k k} T \cong n\left(\varphi(x) e_{i j} T\right),
$$

hence $N_{T}^{*} \varphi(x)=n N_{T}^{*}\left(\varphi(x) e_{i j}\right)$, by Lemma 1.2.

(c) Since $\varphi\left(y_{i j}\right) e_{i j}=e_{i i} y e_{j j}$, it follows from (b) that

$$
N_{R}^{*}\left(y_{i j}\right)=n N_{T}^{*}\left(\varphi\left(y_{i j}\right) e_{i j}\right)=n N_{T}^{*}\left(e_{i i} y e_{j j}\right) \leqslant n N_{T}^{*}(y) .
$$

(d) Using (b) again, we conclude that

$$
\begin{aligned}
N_{T}^{*}(y) & =N_{T}^{*}\left(\sum_{i, j=1}^{n} \varphi\left(y_{i j}\right) e_{i j}\right) \leqslant \sum_{i, j=1}^{n} N_{T}^{*}\left(\varphi\left(y_{i j}\right) e_{i j}\right) \\
& =\sum_{i, j=1}^{n} N_{R}^{*}\left(y_{i j}\right) / n . \quad \square
\end{aligned}
$$

THEOREM 1.8. Every $\aleph_{0}$-continuous regular ring is $N^{*}$-complete.

Proof. For any $\boldsymbol{\aleph}_{0}$-continuous regular ring $R,[4$, Proposition II.11.4] shows that $\operatorname{ker}(\mathbf{P}(R))=0$.

Assume for the moment that $R$ is right and left $\boldsymbol{N}_{0}$-injective. Then the ring $T=M_{2}(R)$ is $\aleph_{0}$-continuous, by [2, Proposition 14.19]. Setting $e=\left(\begin{array}{ll}0 & 0 \\ 0 & 1\end{array}\right)$, we obtain from Lemma 1.6 that $e T(1-e)$ is complete in the $N_{T}^{*}$-metric. There is a group isomorphism $x \mapsto\left(\begin{array}{ll}0 & 0 \\ x & 0\end{array}\right)$ of $R$ onto $e T(1-e)$, and Lemma 1.7 shows that

$$
N_{R}^{*}(x)=2 N_{T}^{*}\left(\left(\begin{array}{ll}
0 & 0 \\
x & 0
\end{array}\right)\right)
$$

for all $x \in R$. Therefore in this case $R$ is $N^{*}$-complete.

In general, let $S$ denote the maximal right $\aleph_{0}$-quotient ring of $R$. Then $S$ is an $\boldsymbol{\aleph}_{0}$-continuous, regular, right and left $\boldsymbol{\aleph}_{0}$-injective overring of $R$, and $R$ contains all the idempotents of $S$ [2, Theorems 14.12 and 14.17]. By the case above, $S$ is $N^{*}$-complete.

Now $R$ and $S$ are unit-regular rings [2, Theorem 14.24], and we may assume they are nonzero. Using Proposition 1.1 and [2, Proposition 14.28], we compute that for any $x \in R$,

$$
\begin{aligned}
N_{R}^{*}(x) & =\inf \left\{k / n \mid k, n \in \mathbf{N} \text { and } n(x R) \lesssim k R_{R}\right\} \\
& =\inf \left\{k / n \mid k, n \in \mathbf{N} \text { and } n(x S) \lesssim k S_{S}\right\}=N_{S}^{*}(x) .
\end{aligned}
$$

Consequently, the $N^{*}$-completeness of $R$ will follow from the $N^{*}$-completeness of $S$ provided $R$ is closed in $S$ in the $N_{S}^{*}$-metric.

We claim that $N_{S}^{*}(x)=1$ for any $x \in S-R$. By [2, Proposition 3.15], $S$ has a two-sided ideal $N$ such that $N \subseteq R$ and the ring $S / N$ is abelian. Then $x \notin N$, hence $S$ has a primitive ideal $M$ such that $N \subseteq M$ but $x \notin M$. As $S / N$ is abelian, $S / M$ is a division ring, hence $S / M$ has a unique rank function $Q$ and $Q(x+M)=1$. Pulling $Q$ back to a pseudo-rank function $P$ on $S$, we obtain $P(x)=1$, so that $N_{S}^{*}(x) \geqslant 1$. Thus $N_{S}^{*}(x)=1$, as claimed. 
As a result, $N_{S}^{*}(x-y)=1$ for all $x \in S-R$ and all $y \in R$, whence $R$ is closed in $S$ in the $N_{S}^{*}$-metric, as desired. Therefore $R$ is $N^{*}$-complete.

COROLlARY 1.9. Every regular, right and left self-injective ring is $N^{*}$-complete.

We conclude this section by proving that $N^{*}$-completeness carries over to matrix rings and certain factor rings.

THEOREM 1.10. If $R$ is an $N^{*}$-complete regular ring, then any matrix ring $M_{n}(R)$ is $N^{*}$-complete.

Proof. Set $T=M_{n}(R)$, let $\varphi: R \rightarrow T$ be the natural map, and let $\left\{e_{i j} \mid i, j=\right.$ $1, \ldots, n\}$ be the standard matrix units in $T$. Given any $y \in \operatorname{ker}(\mathbf{P}(T))$, we have $N_{T}^{*}(y)=0$, whence Lemma 1.7 shows that $N_{R}^{*}\left(y_{i j}\right)=0$ for all $i, j$. Then all $y_{i j}=0$, so that $y=0$. Thus $\operatorname{ker}(\mathbf{P}(T))=0$.

Now consider any sequence $\left\{y^{(1)}, y^{(2)}, \ldots\right\}$ in $T$ that is Cauchy with respect to $N_{T}^{*}$. Fixing $i$ and $j$ for a while, we have

$$
N_{R}^{*}\left(y_{i j}^{(k)}-y_{i j}^{(l)}\right) \leqslant n N_{T}^{*}\left(y^{(k)}-y^{(l)}\right)
$$

for all $k, l$ (Lemma 1.7), hence the sequence $\left\{y_{i j}^{(1)}, y_{i j}^{(2)}, \ldots\right\}$ in $R$ is Cauchy with respect to $N_{R}^{*}$. Consequently, there exists $y_{i j} \in R$ such that $y_{i j}^{(k)} \rightarrow y_{i j}$ in the $N_{R}^{*}$ metric. Having gotten such elements $y_{i j}$ for each $i, j$, we obtain a matrix $y \in T$ with entries $y_{i j}$. Inasmuch as

$$
N_{T}^{*}\left(y^{(k)}-y\right) \leqslant \sum_{i, j=1}^{n} N_{R}^{*}\left(y_{i j}^{(k)}-y_{i j}\right) / n
$$

for all $k$ (Lemma 1.7 again), we conclude that $y^{(k)} \rightarrow y$ in the $N_{T}^{*}$-metric.

Therefore $T$ is $N^{*}$-complete.

LEMma 1.11. Let $J$ be a two-sided ideal in a regular ring $R$, let $A$ and $B$ be finitely generated projective right $R$-modules, and let $n \in \mathbf{N}$. If $n(A / A J) \lesssim B / B J$, then there exists a decomposition $A=A^{\prime} \oplus A^{\prime \prime}$ such that $n A^{\prime} \lesssim B$ and $A^{\prime \prime}=A^{\prime \prime} J$.

Proof. As $(n A) /(n A) J \lesssim B / B J$, we may apply [2, Proposition 2.20] to obtain a decomposition $n A=C \oplus D$ such that $C \lesssim B$ and $D=D J$. Then, by [2, Theorem 2.8], there exist decompositions $C=C_{1} \oplus \cdots \oplus C_{n}$ and $D=D_{1} \oplus \cdots \oplus D_{n}$ such that $C_{i} \oplus D_{i} \cong A$ for all $i$. Since $D=D J$, each $D_{i}=D_{i} J$, hence $C_{i} / C_{i} J \cong A / A J$. Thus the modules $C_{i} / C_{i} J$ are pairwise isomorphic, so by [2, Proposition 2.19] there exist decompositions $C_{i}=E_{i} \oplus F_{i}$ for each $i$ such that the $E_{i}$ are pairwise isomorphic and each $F_{i}=F_{i} J$.

Now $A \cong C_{1} \oplus D_{1}=E_{1} \oplus F_{1} \oplus D_{1}$, so there is a decomposition $A=A^{\prime} \oplus A^{\prime \prime}$ with $A^{\prime} \cong E_{1}$ and $A^{\prime \prime} \cong F_{1} \oplus D_{1}$. Then

$$
n A^{\prime} \cong n E_{1} \cong E_{1} \oplus \cdots \oplus E_{n} \leqslant C_{1} \oplus \cdots \oplus C_{n}=C \lesssim B
$$

Since $D_{1}=D_{1} J$ and $F_{1}=F_{1} J$, we also have $A^{\prime \prime}=A^{\prime \prime} J$.

For the moment, we now restrict to unit-regular rings. This restriction will be removed when we prove that all $N^{*}$-complete regular rings are unit-regular (Theorem 2.3). 
LEMMA 1.12. Let $J$ be a two-sided ideal in a unit-regular ring $R$. Then

$$
N_{R / J}^{*}(x+J)=\inf \left\{N_{R}^{*}(y) \mid y \in x+J\right\}
$$

for all $x \in R$.

Proof. This is clear if $J=R$, so assume $J \neq R$.

Given any $y \in x+J$, we see that

$$
\begin{aligned}
N_{R / J}^{*}(x+J) & =N_{R / J}^{*}(y+J)=\sup \{Q(y+J) \mid Q \in \mathbf{P}(R / J)\} \\
& =\sup \{P(y) \mid P \in \mathbf{P}(R) \text { and } J \subseteq \operatorname{ker}(P)\} \\
& \leqslant \sup \{P(y) \mid P \in \mathbf{P}(R)\}=N_{R}^{*}(y) .
\end{aligned}
$$

Thus $N_{R / J}^{*}(x+J) \leqslant \inf \left\{N_{R}^{*}(y) \mid y \in x+J\right\}$.

Given any real number $\alpha>N_{R / J}^{*}(x+J)$, Proposition 1.1 shows that there exist $k, n \in \mathbf{N}$ for which $k / n<\alpha$ and

$$
n((x+J)(R / J)) \lesssim k(R / J)
$$

that is, $n(x R / x J) \lesssim(k R) /(k R) J$. According to Lemma 1.11, there is a decomposition $x R=A^{\prime} \oplus A^{\prime \prime}$ such that $n A^{\prime} \lesssim k R_{R}$ and $A^{\prime \prime}=A^{\prime \prime} J$. Then $x=y+z$ for some $y \in A^{\prime}$ and $z \in A^{\prime \prime}$. Note that $z \in J$, so that $y \in x+J$. Since $n(y R) \leqslant n A^{\prime} \lesssim k R_{R}$, we conclude from Proposition 1.1 that $N_{R}^{*}(y) \leqslant k / n<\alpha$. Therefore

$$
\inf \left\{N_{R}^{*}(y) \mid y \in x+J\right\} \leqslant N_{R / J}^{*}(x+J) .
$$

THEOREM 1.13. Let $R$ be an $N^{*}$-complete unit-regular ring, and let $J$ be a two-sided ideal of $R$. Then the following conditions'are equivalent.

(a) $R / J$ is $N^{*}$-complete.

(b) $J$ is $N^{*}$-closed in $R$.

(c) $J=\operatorname{ker}(X)$ for some $X \subseteq \mathbf{P}(R)$.

Proof. (a) $\Rightarrow$ (c): By definition, $\operatorname{ker}(\mathbf{P}(R / J))=0$, hence if

$$
X=\{P \in \mathbf{P}(R) \mid J \subseteq \operatorname{ker}(P)\},
$$

then $J=\operatorname{ker}(X)$.

(c) $\Rightarrow$ (b): Given $x \in R-J$, we must have $P(x)>0$ for some $P \in X$. Then

$$
N^{*}(y-x) \geqslant P(y-x) \geqslant P(x)-P(y)=P(x)
$$

for all $y \in J$, hence $x$ is not in the $N^{*}$-closure of $J$. Thus $J$ is $N^{*}$-closed.

(b) $\Rightarrow$ (a): Given a nonzero coset $x+J$ in $R / J$, we have $x \notin J$, hence there must exist a positive real number $\varepsilon$ such that $N_{R}^{*}(y-x) \geqslant \varepsilon$ for all $y \in J$. Then $N_{R}^{*}(z) \geqslant \varepsilon$ for all $z \in x+J$, whence $N_{R / J}^{*}(x+J) \geqslant \varepsilon$, by Lemma 1.12. Consequently, $\operatorname{ker}(\mathbf{P}(R / J))=0$.

Now consider a sequence $\left\{a_{1}, a_{2}, \ldots\right\}$ in $R / J$ that is Cauchy with respect to $N_{R / J}^{*}$. There is no loss of generality in assuming that $N_{R / J}^{*}\left(a_{n+1}-a_{n}\right)<1 / 2^{n}$ for all $n$. Choose $x_{1}, x_{2}, \ldots$ in $R$ such that each $a_{n}=x_{n}+J$.

Set $y_{1}=x_{1}$. Then $N_{R / J}^{*}\left(\left(x_{2}-y_{1}\right)+J\right)<1 / 2$, so by Lemma 1.12 there exists $z$ in $\left(x_{2}-y_{1}\right)+J$ satisfying $N_{R}^{*}(z)<1 / 2$. Set $y_{2}=y_{1}+z$, so that $y_{2}+J=x_{2}+J=a_{2}$ and $N_{R}^{*}\left(y_{2}-y_{1}\right)<1 / 2$. Continuing in this manner, we obtain elements $y_{1}, y_{2}, \ldots$ in $R$ such that $y_{n}+J=a_{n}$ and $N_{R}^{*}\left(y_{n+1}-y_{n}\right)<1 / 2^{n}$ for all $n$. 
Thus $\left\{y_{1}, y_{2}, \ldots\right\}$ is Cauchy with respect to $N_{R}^{*}$, hence there exists $y \in R$ such that $y_{n} \rightarrow y$ in the $N_{R}^{*}$-metric. Setting $a=y+J$, we conclude from Lemma 1.12 that $a_{n} \rightarrow a$ in the $N_{R / J}^{*}$-metric.

Therefore $R / J$ is $N^{*}$-complete.

COROLlARY 1.14. Let $R$ be an $N^{*}$-complete unit-regular ring, and let $M$ be $a$ maximal two-sided ideal of $R$. Then $R / M$ is $N^{*}$-complete.

Proof. As $R / M$ is a simple unit-regular ring, [2, Corollary 18.5] shows that there is a rank function on $R / M$. Then there exists $P \in \mathbf{P}(R)$ such that $\operatorname{ker}(P)=M$, hence $R / M$ is $N^{*}$-complete by Theorem 1.13 .

II. $K_{0}$. A good deal of information about a regular ring $R$, particularly ideal theory and decomposition properties of the principal right ideals, is stored in the Grothendieck group $K_{0}(R)$. We study this group for an $N^{*}$-complete regular ring $R$ in this section, proving that $K_{0}(R)$ is an archimedean, norm-complete, partially ordered abelian group with the interpolation property. In the following section, we develop a structure theory for such groups, which can then be applied, via $K_{0}$, to the structure theory of $N^{*}$-complete regular rings.

Definition. Recall that the Grothendieck group $K_{0}$ of a ring $R$ is an abelian group with generators $[A]$ corresponding to the finitely generated projective right $R$-modules $A$ and with relations $[A]+[B]=[C]$ whenever $A \oplus B \cong C$. All elements of $K_{0}(R)$ are of the form $[A]-[B]$, for suitable $A$ and $B$. We set

$$
K_{0}(R)^{+}=\{[A] \mid A \text { is a finitely generated projective right } R \text {-module }\},
$$

and we define a relation $\leqslant$ on $K_{0}(R)$ so that $x \leqslant y$ if and only if $y-x$ lies in $K_{0}(R)^{+}$. This relation is a translation-invariant pre-order on $K_{0}(R)$, so that $K_{0}(R)$ becomes a pre-ordered abelian group. The element $[R]$ is an order-unit in $K_{0}(R)$, meaning that for any $x \in K_{0}(R)$ there exists $n \in \mathbf{N}$ such that $x \leqslant n[R]$.

For a unit-regular ring $R$, the relations between $K_{0}(R)$ and the finitely generated projective right $R$-modules are much cleaner than in general. Namely, for any finitely generated projective right $R$-modules $A, B, C, D$ we have

$$
\begin{aligned}
& {[A]-[B]=[C]-[D] \text { if and only if } A \oplus D \cong B \oplus C,} \\
& {[A]-[B] \leqslant[C]-[D] \text { if and only if } A \oplus D \lesssim B \oplus C}
\end{aligned}
$$

[2, Proposition 15.2]. In addition, the relation $\leqslant$ on $K_{0}(R)$ is actually a partial order, so that $K_{0}(R)$ is a partially ordered abelian group in this case. Thus, in order to deal effectively with $K_{0}$ of $N^{*}$-complete regular rings, we first prove that such rings are unit-regular. Two lemmas will be helpful in doing this.

LemMA 2.1. Let $R$ be an $N^{*}$-complete regular ring, and let $A, B, C$ be finitely generated projective right $R$-modules. Let $\left\{A_{1}, A_{2}, \ldots\right\}$ and $\left\{B_{1}, B_{2}, \ldots\right\}$ be independent sequences of finitely generated submodules of $A$ and $B$, such that $A_{k} \cong B_{k}$ for all $k$. For each $k$, let $A_{k}^{*}$ be a submodule of $A$ such that

$$
A=A_{1} \oplus \cdots \oplus A_{k} \oplus A_{k}^{*},
$$

and assume that $2^{k} t_{k} A_{k}^{*} \lesssim t_{k} C$ for some $t_{k} \in \mathbf{N}$. Then $A \lesssim B$. 
Proof. As all matrix rings over $R$ are $N^{*}$-complete (Theorem 1.10), we may use the standard Morita-equivalences to transfer our problem to the category of right modules over a suitable matrix ring $M_{n}(R)$, with $n$ chosen large enough so that the modules corresponding to $A, B, C$ are cyclic. Thus there is no loss of generality in assuming that $A, B, C$ are actually principal right ideals of $R$.

Choose idempotents $e, f \in R$ such that $e R=A$ and $f R=B$. Applying [2, Proposition 2.13] to the ascending sequence

$$
A_{1} \leqslant A_{1} \oplus A_{2} \leqslant A_{1} \oplus A_{2} \oplus A_{3} \leqslant \ldots
$$

of finitely generated submodules of $A$, we obtain orthogonal idempotents $e_{1}, e_{2}, \ldots$ in $e R e$ such that

$$
e_{1} R \oplus \cdots \oplus e_{k} R=A_{1} \oplus \cdots \oplus A_{k}
$$

for all $k$. Similarly, there exist orthogonal idempotents $f_{1}, f_{2}, \ldots$ in $f R f$ such that

$$
f_{1} R \oplus \cdots \oplus f_{k} R=B_{1} \oplus \cdots \oplus B_{k}
$$

for all $k$. Note that each

$$
\begin{aligned}
e_{k} R & \cong\left(e_{1} R \oplus \cdots \oplus e_{k} R\right) /\left(e_{1} R \oplus \cdots \oplus e_{k-1} R\right) \\
& =\left(A_{1} \oplus \cdots \oplus A_{k}\right) /\left(A_{1} \oplus \cdots \oplus A_{k-1}\right) \cong A_{k}
\end{aligned}
$$

and similarly $f_{k} R \cong B_{k}$, so that $e_{k} R \cong f_{k} R$. Thus there exist elements $x_{k} \in e_{k} R f_{k}$ and $y_{k} \in f_{k} R e_{k}$ such that $x_{k} y_{k}=e_{k}$ and $y_{k} x_{k}=f_{k}$.

For each $k$, we have $\left(e-e_{1}-\cdots-e_{k}\right) R \cong A_{k}^{*}$, whence

$$
2^{k} t_{k}\left(\left(e-e_{1}-\cdots-e_{k}\right) R\right) \lesssim t_{k} C \lesssim t_{k} R_{R}
$$

and consequently $N^{*}\left(e-e_{1}-\cdots-e_{k}\right) \leqslant 1 / 2^{k}$, by Lemma 1.2. Thus $\Sigma e_{k} \rightarrow e$ in the $N^{*}$-metric. As

$$
x_{k+1}=e_{k+1} x_{k+1}=\left(e-e_{1}-\cdots-e_{k}\right) e_{k+1} x_{k+1}
$$

for each $k$, we also obtain $N^{*}\left(x_{k+1}\right) \leqslant 1 / 2^{k}$, and similarly, $N^{*}\left(y_{k+1}\right) \leqslant 1 / 2^{k}$.

Now the partial sums of the series $\Sigma x_{k}$ and $\Sigma y_{k}$ are Cauchy with respect to $N^{*}$, hence there exist $x, y \in R$ such that $\Sigma x_{k} \rightarrow x$ and $\Sigma y_{k} \rightarrow y$ in the $N^{*}$-metric. As each

$$
x_{k}=e_{k} x_{k} f_{k}=e e_{k} x_{k} f_{k} f=e x_{k} f,
$$

we obtain $x=\operatorname{exf}$, and likewise $y=f y e$. Since $x_{i} y_{j}=x_{i} f_{i} f_{j} y_{j}=0$ whenever $i \neq j$, we have

$$
\left(x_{1}+\cdots+x_{k}\right)\left(y_{1}+\cdots+y_{k}\right)=x_{1} y_{1}+\cdots+x_{k} y_{k}=e_{1}+\cdots+e_{k}
$$

for all $k$, and consequently $x y=e$. Therefore $e R \lesssim f R$, that is, $A \lesssim B$.

LEMMA 2.2. Let $A, B, C$ be finitely generated projective right modules over a regular ring, such that $A \oplus C \cong B \oplus C$. Then there exist decompositions

$$
A=A^{\prime} \oplus A^{\prime \prime} ; \quad B=B^{\prime} \oplus B^{\prime \prime} ; \quad C=C^{\prime} \oplus C^{\prime \prime}
$$

such that $A^{\prime} \cong B^{\prime}$ and $A^{\prime \prime} \cong C^{\prime}$, while also $A^{\prime \prime} \oplus C^{\prime \prime} \cong B^{\prime \prime} \oplus C^{\prime \prime}$. 
Proof. According to [2, Theorem 2.8], there exist decompositions $A=A^{\prime} \oplus A^{\prime \prime}$ and $C=D \oplus E$ such that $A^{\prime} \oplus D \cong B$ and $A^{\prime \prime} \oplus E \cong C$. Then we obtain decompositions $B=B^{\prime} \oplus B^{\prime \prime}$ and $C=C^{\prime} \oplus C^{\prime \prime}$ such that $B^{\prime} \cong A^{\prime}$ and $B^{\prime \prime} \cong D$, while also $C^{\prime} \cong A^{\prime \prime}$ and $C^{\prime \prime} \cong E$. Finally,

$$
A^{\prime \prime} \oplus C^{\prime \prime} \cong A^{\prime \prime} \oplus E \cong C=D \oplus E \cong B^{\prime \prime} \oplus C^{\prime \prime}
$$

THEOREM 2.3. Every $N^{*}$-complete regular ring is unit-regular.

Proof. Given an $N^{*}$-complete regular ring $R$, we have $\operatorname{ker}(\mathbf{P}(R))=0$ by definition, hence all matrix rings over $R$ are directly finite [2, Proposition 16.11]. To prove that $R$ is unit-regular, it suffices to show that if $A, B, C$ are any finitely generated projective right $R$-modules satisfying $A \oplus C \cong B \oplus C$, then $A \cong B$.

Inducting on Lemma 2.2, we obtain submodules

$$
A_{1}^{\prime}, A_{1}^{\prime \prime}, A_{2}^{\prime}, A_{2}^{\prime \prime}, \ldots \leqslant A ; \quad B_{1}^{\prime}, B_{1}^{\prime \prime}, B_{2}^{\prime}, B_{2}^{\prime \prime}, \ldots \leqslant B ; \quad C_{1}^{\prime}, C_{1}^{\prime \prime}, C_{2}^{\prime}, C_{2}^{\prime \prime}, \ldots \leqslant C
$$

such that

$$
A=A_{1}^{\prime} \oplus A_{1}^{\prime \prime} ; \quad B=B_{1}^{\prime} \oplus B_{1}^{\prime \prime} ; \quad C=C_{1}^{\prime} \oplus C_{1}^{\prime \prime}
$$

while also

$$
\begin{gathered}
A_{i}^{\prime \prime}=A_{i+1}^{\prime} \oplus A_{i+1}^{\prime \prime} ; \quad B_{i}^{\prime \prime}=B_{i+1}^{\prime} \oplus B_{i+1}^{\prime \prime} ; \quad C_{i}^{\prime \prime}=C_{i+1}^{\prime} \oplus C_{i+1}^{\prime \prime} \\
A_{i}^{\prime} \cong B_{i}^{\prime} ; \quad A_{i}^{\prime \prime} \cong C_{i}^{\prime} ; \quad A_{i}^{\prime \prime} \oplus C_{i}^{\prime \prime} \cong B_{i}^{\prime \prime} \oplus C_{i}^{\prime \prime}
\end{gathered}
$$

for all $i$. Note that $A_{1}^{\prime \prime} \geqslant A_{2}^{\prime \prime} \geqslant \ldots$, and that $C_{1}^{\prime}, C_{2}^{\prime}, \ldots$ are independent submodules of $C$. Consequently, we obtain

$$
i A_{i}^{\prime \prime} \lesssim A_{1}^{\prime \prime} \oplus A_{2}^{\prime \prime} \oplus \cdots \oplus A_{i}^{\prime \prime} \cong C_{1}^{\prime} \oplus C_{2}^{\prime} \oplus \cdots \oplus C_{i}^{\prime} \leqslant C
$$

for all $i$.

Now set $A_{1}=A_{1}^{\prime} \oplus A_{2}^{\prime}$ and $B_{1}=B_{1}^{\prime} \oplus B_{2}^{\prime}$, while $A_{1}^{*}=A_{2}^{\prime \prime}$. Set

$$
A_{k}=\bigoplus_{i=2^{k-1}+1}^{2^{k}} A_{i}^{\prime} ; \quad A_{k}^{*}=A_{2^{k}}^{\prime \prime} ; \quad B_{k}=\bigoplus_{i=2^{k-1}+1}^{2^{k}} B_{i}^{\prime}
$$

for all $k=2,3, \ldots$ Thus $\left\{A_{1}, A_{2}, \ldots\right\}$ and $\left\{B_{1}, B_{2}, \ldots\right\}$ are independent sequences of finitely generated submodules of $A$ and $B$, with $A_{k} \cong B_{k}$ for all $k$. Also, since

$$
A=A_{1}^{\prime} \oplus A_{1}^{\prime \prime}=A_{1}^{\prime} \oplus A_{2}^{\prime} \oplus A_{2}^{\prime \prime}=\cdots=A_{1}^{\prime} \oplus A_{2}^{\prime} \oplus \cdots \oplus A_{i}^{\prime} \oplus A_{i}^{\prime \prime}
$$

for all $i$, we have $A=A_{1} \oplus A_{2} \oplus \cdots \oplus A_{k} \oplus A_{k}^{*}$ for all $k$. Inasmuch as $2^{k} A_{k}^{*} \lesssim C$ for all $k$, we conclude from Lemma 2.1 that $A \lesssim B$.

By symmetry, $B \lesssim A$. As all matrix rings over $R$ are directly finite, it follows that $A \cong B$ [2, Proposition 5.4].

Definition. Let $G$ be a partially ordered abelian group. Then $G$ is said to be an interpolation group if given any $x_{1}, x_{2}, y_{1}, y_{2}$ in $G$ satisfying $x_{i} \leqslant y_{j}$ for all $i, j$, there exists $z \in G$ such that $x_{i} \leqslant z \leqslant y_{j}$ for all $i, j$. Equivalently, $G$ is an interpolation group if and only if either of the following forms of the Riesz decomposition property holds.

(a) If $x, y_{1}, \ldots, y_{n} \in G^{+}$and $x \leqslant y_{1}+\cdots+y_{n}$, then there exist $x_{1}, \ldots, x_{n}$ in $G^{+}$ such that $x=x_{1}+\cdots+x_{n}$ and each $x_{i} \leqslant y_{i}$. 
(b) If $x_{1}, \ldots, x_{n}, y_{1}, \ldots, y_{k} \in G^{+}$and $x_{1}+\cdots+x_{n}=y_{1}+\cdots+y_{k}$, then there exist $z_{i j} \in G^{+}$(for $i=1, \ldots, n$ and $j=1, \ldots, k$ ) such that $x_{i}=z_{i 1}+\cdots+z_{i k}$ for all $i$ and $y_{j}=z_{\mathrm{l} j}+\cdots+z_{n j}$ for all $j$.

For any unit-regular ring $R$, the partially ordered abelian group $K_{0}(R)$ is an interpolation group [4, Proposition II.10.3]. In particular, this holds for any $N^{*}$-complete regular ring, because of Theorem 2.3; however, we postpone recording this fact until Theorem 2.11.

Definition. Let $G$ be a partially ordered abelian group, and let $n$ be a positive integer. We say that $G$ is $n$-unperforated if for all $x \in G$, we have $n x \geqslant 0$ only when $x \geqslant 0$. If $G$ is $n$-unperforated for all $n \in \mathbf{N}$, then $G$ is said to be unperforated. The group $G$ is archimedean provided that whenever $x, y \in G$ and $n x \leqslant y$ for all $n \in \mathbf{N}$, then $x \leqslant 0$. It is easily checked that all archimedean directed abelian groups are unperforated [4, Lemma I.5.2]. Our next goal is to prove that $K_{0}$ of any $N^{*}$-complete regular ring $R$ is archimedean; however, since our proof requires $K_{0}(R)$ to be 2-unperforated, we show that first.

Lemma 2.4. Let $A$ and $B$ be finitely generated projective right modules over a unit-regular ring $R$, and let $n \in \mathbf{N}$. If $A \lesssim n B$, then there exists a decomposition $A=A^{\prime} \oplus A^{\prime \prime}$ such that $A^{\prime} \lesssim B$ and $n A^{\prime \prime} \lesssim(n-1) A$.

Proof. Since $A \lesssim n B$, there is a decomposition $A=A_{1} \oplus \cdots \oplus A_{n}$ with each $A_{i} \lesssim B$ [2, Corollary 2.9]. Each $A_{i}$ is isomorphic to a submodule $B_{i} \leqslant B$, and we define $B^{\prime}=B_{1}+\cdots+B_{n}$. Then $B^{\prime}$ is a finitely generated submodule of $B$, and

$$
B^{\prime} \lesssim B_{1} \oplus \cdots \oplus B_{n} \cong A_{1} \oplus \cdots \oplus A_{n}=A,
$$

hence we obtain a decomposition $A=A^{\prime} \oplus A^{\prime \prime}$ with $A^{\prime} \cong B^{\prime} \leqslant B$. For each $j=$ $1, \ldots, n$, we have $A_{j} \cong B_{j} \leqslant B^{\prime} \cong A^{\prime}$ and so

$$
A^{\prime} \oplus A^{\prime \prime}=A=A_{1} \oplus \cdots \oplus A_{n} \lesssim A^{\prime} \oplus\left(\bigoplus_{i \neq j} A_{i}\right),
$$

whence $A^{\prime \prime} \lesssim \bigoplus_{i \neq j} A_{i}$. Therefore

$$
n A^{\prime \prime} \lesssim \bigoplus_{j=1}^{n} \bigoplus_{i \neq j} A_{i} \cong \bigoplus_{i=1}^{n}(n-1) A_{i} \cong(n-1) A .
$$

Lemma 2.5. Let $A$ and $B$ be finitely generated projective right modules over a unit-regular ring $R$, and let $n \in \mathbf{N}$. If $n A \lesssim n B$, then there exist decompositions $A=A^{\prime} \oplus A^{\prime \prime}$ and $B=B^{\prime} \oplus B^{\prime \prime}$ such that $A^{\prime} \cong B^{\prime}$ and $n A^{\prime \prime} \lesssim n B^{\prime \prime}$, while also $2 t A^{\prime \prime} \lesssim$ $t A$ for some $t \in \mathbf{N}$.

Proof. By Lemma 2.4, there exist decompositions $A=A_{1} \oplus A_{1}^{*}$ and $B=B_{1} \oplus B_{1}^{*}$ such that $A_{1} \cong B_{1}$ and $n A_{1}^{*} \lesssim(n-1) A$. Since

$$
n A_{1} \oplus n A_{1}^{*} \cong n A \lesssim n B=n B_{1} \oplus n B_{1}^{*} \cong n A_{1} \oplus n B_{1}^{*},
$$

we also have $n A_{1}^{*} \lesssim n B_{1}^{*}$. Thus we may continue by induction, obtaining submodules

$$
A_{1}, A_{1}^{*}, A_{2}, A_{2}^{*}, \ldots \leqslant A ; \quad B_{1}, B_{1}^{*}, B_{2}, B_{2}^{*}, \ldots \leqslant B
$$


such that

$$
\begin{gathered}
A_{i}^{*}=A_{i+1} \oplus A_{i+1}^{*} ; \quad B_{i}^{*}=B_{i+1} \oplus B_{i+1}^{*} ; \\
A_{i+1} \cong B_{i+1} ; \quad n A_{i+1}^{*} \lesssim(n-1) A_{i}^{*} ; \quad n A_{i+1}^{*} \lesssim n B_{i+1}^{*}
\end{gathered}
$$

for all $i$. In addition,

$$
n^{i} A_{i}^{*} \lesssim n^{i-1}(n-1) A_{i-1}^{*} \lesssim \cdots \lesssim n(n-1)^{i-1} A_{1}^{*} \lesssim(n-1)^{i} A
$$

for all $i$.

Choose $i \in \mathrm{N}$ such that $((n-1) / n)^{i} \leqslant 1 / 2$, and set $t=(n-1)^{i}$, so that $2 t \leqslant n^{i}$. Setting $A^{\prime}=A_{1} \oplus \cdots \oplus A_{i}$ and $A^{\prime \prime}=A_{i}^{*}$, we obtain $A=A^{\prime} \oplus A^{\prime \prime}$ and

$$
2 t A^{\prime \prime} \lesssim n^{i} A_{i}^{*} \lesssim(n-1)^{i} A=t A .
$$

Setting $B^{\prime}=B_{1} \oplus \cdots \oplus B_{i}$ and $B^{\prime \prime}=B_{i}^{*}$, we obtain $B=B^{\prime} \oplus B^{\prime \prime}$, while also $A^{\prime} \cong B^{\prime}$ and $n A^{\prime \prime} \lesssim n B^{\prime \prime}$.

THEOREM 2.6. Let $R$ be an $N^{*}$-complete regular ring, let $A$ and $B$ be finitely generated projective right $R$-modules, and let $n \in \mathbf{N}$. If $n A \lesssim n B$, then $A \lesssim B$.

Proof. Inducting on Lemma 2.5, we obtain submodules

$$
A_{1}, A_{1}^{*}, A_{2}, A_{2}^{*}, \ldots \leqslant A ; \quad B_{1}, B_{1}^{*}, B_{2}, B_{2}^{*}, \ldots \leqslant B
$$

such that $A=A_{1} \oplus A_{1}^{*}$ and $B=B_{1} \oplus B_{1}^{*}$, while also

$$
\begin{aligned}
& A_{k}^{*}=A_{k+1} \oplus A_{k+1}^{*} ; \quad B_{k}^{*}=B_{k+1} \oplus B_{k+1}^{*} ; \\
& A_{k} \cong B_{k} ; \quad n A_{k}^{*} \lesssim n B_{k}^{*} ; \quad 2^{k} t_{k} A_{k}^{*} \lesssim t_{k} A
\end{aligned}
$$

(for some $t_{k} \in \mathbf{N}$ ) for all $k$. Applying Lemma 2.1, we conclude that $A \lesssim B$.

As an $N^{*}$-complete regular ring $R$ is unit-regular, it follows immediately from Theorem 2.6 that $K_{0}(R)$ is unperforated. This result will be subsumed by the stronger result that $K_{0}(R)$ is archimedean (Theorem 2.11).

LEMMA 2.7. Let $R$ be an $N^{*}$-complete regular ring, let $A, B, C$ be finitely generated projective right $R$-modules, and let $n \in \mathbf{N}$. If $2^{n} A \lesssim 2^{n} B \oplus C$, then there exists $a$ decomposition $A=A^{\prime} \oplus A^{\prime \prime}$ such that $A^{\prime} \lesssim B$ and $2^{n} A^{\prime \prime} \lesssim C$.

Proof. The proof of [2, Lemma 14.31] may be used, substituting Theorem 2.6 for [2, Theorem 14.30].

THEOREM 2.8. Let $R$ be an $N^{*}$-complete regular ring, and let $A, B, C$ be finitely generated projective right $R$-modules. If $2^{n} A \lesssim 2^{n} B \oplus C$ for all $n \in \mathbf{N}$, then $A \lesssim B$.

Proof. Since $2 A \lesssim 2 B \oplus C$, Lemma 2.7 provides us with decompositions $A=$ $A_{1} \oplus A_{1}^{*}$ and $B=B_{1} \oplus B_{1}^{*}$ such that $A_{1} \cong B_{1}$ and $2 A_{1}^{*} \lesssim C$. For all $n \in \mathbf{N}$,

$$
2^{n} A_{1} \oplus 2^{n} A_{1}^{*} \cong 2^{n} A \lesssim 2^{n} B \oplus C \cong 2^{n} B_{1} \oplus 2^{n} B_{1}^{*} \oplus C \cong 2^{n} A_{1} \oplus 2^{n} B_{1}^{*} \oplus C,
$$

hence $2^{n} A_{1}^{*} \lesssim 2^{n} B_{1}^{*} \oplus C$. Thus we may continue by induction, obtaining submodules

$$
A_{1}, A_{1}^{*}, A_{2}, A_{2}^{*}, \ldots \leqslant A ; \quad B_{1}, B_{1}^{*}, B_{2}, B_{2}^{*}, \ldots \leqslant B
$$


such that

$$
\begin{gathered}
A_{k}^{*}=A_{k+1} \oplus A_{k+1}^{*} ; \quad B_{k}^{*}=B_{k+1} \oplus B_{k+1}^{*} ; \\
A_{k} \cong B_{k} ; \quad 2^{k} A_{k}^{*} \lesssim C ; \quad 2^{n} A_{k}^{*} \lesssim 2^{n} B_{k}^{*} \oplus C
\end{gathered}
$$

for all $k, n$. Applying Lemma 2.1 , we conclude that $A \lesssim B$.

It follows directly from Theorem 2.8 that $K_{0}$ of any $N^{*}$-complete regular ring is archimedean. We postpone recording this result until Theorem 2.11.

Definition. Let $(G, u)$ be a partially ordered abelian group with order-unit. For any $x \in G$, we define

$$
\|x\|_{u}=\inf \{k / n \mid k, n \in \mathbf{N} \text { and }-k u \leqslant n x \leqslant k u\},
$$

and we note that $\|x\|_{u}$ is a nonnegative real number. When there is no danger of confusion as to the order-unit $u$, we just write $\|x\|$ instead of $\|x\|_{u}$. The function $\|\cdot\|$ behaves like a seminorm on $G$, for $\|m x\|=|m| \cdot\|x\|$ and $\|x+y\| \leqslant\|x\|+$ $\|y\|$ for all $x, y \in G$ and $m \in \mathbf{Z}$ [4, Lemma I.6.1]. In particular, it follows that the rule $\delta(x, y)=\|x-y\|$ defines a pseudo-metric $\delta$ on $G$. If $\delta$ is actually a metric, and $G$ is complete in this metric, then we say that $(G, u)$ is norm-complete.

It is tempting to expect norm-complete partially ordered abelian groups with order-unit, particularly those that are interpolation groups, to be archimedean, but this is not the case in general. For instance, make the group $G=\mathbf{R}^{2}$ into a partially ordered abelian group with positive cone

$$
G^{+}=\{(0,0)\} \cup\{(a, b) \in G \mid a>0 \text { and } b>0\} .
$$

It is clear that $G$ is an interpolation group, and that the element $u=(1,1)$ is an order-unit in $G$. Observing that

$$
\|(a, b)\|_{u}=\max \{|a|,|b|\}
$$

for all $(a, b) \in G$, we see that $(G, u)$ is norm-complete. However, $n(-1,0) \leqslant u$ for all $n \in \mathbf{N}$ while $(-1,0) \$ 0$, so that $G$ is not archimedean.

LEMMA 2.9. Let $R$ be an $N^{*}$-complete regular ring, let $v \in K_{0}(R)$, and let $n \in \mathbf{N}$. If $\|v\|<1 / 2^{n}$, then there exist $x, y \in R$ such that $v=[x R]-[y R]$, while also $N^{*}(x)$ $\leqslant 1 / 2^{n}$ and $N^{*}(y) \leqslant 1 / 2^{n}$.

Proof. Write $v=[A]-[B]$ for some finitely generated projective right $R$-modules $A$ and $B$. Since $\|v\|<1 / 2^{n}$, there exist $s, t \in \mathbf{N}$ such that $-s[R] \leqslant t v \leqslant s[R]$ and $s / t<1 / 2^{n}$. Since $R$ is unit-regular (Theorem 2.3), it follows that

$$
t A \lesssim s R_{R} \oplus t B \quad \text { and } \quad t B \lesssim s R_{R} \oplus t A .
$$

Now $2^{n} t A \lesssim 2^{n} t B \oplus t R_{R}$, because $2^{n} s<t$. Then $2^{n} A \lesssim 2^{n} B \oplus R_{R}$ by Theorem 2.6, and similarly $2^{n} B \lesssim 2^{n} A \oplus R_{R}$.

In view of Lemma 2.7, there exist decompositions $A=A_{1} \oplus A_{2}$ and $B=B_{1} \oplus B_{2}$ such that $A_{1} \cong B_{1}$ and $2^{n} A_{2} \lesssim R_{R}$. Then

$$
2^{n} B_{1} \oplus 2^{n} B_{2} \cong 2^{n} B \lesssim 2^{n} A \oplus R_{R} \cong 2^{n} A_{1} \oplus 2^{n} A_{2} \oplus R_{R} \cong 2^{n} B_{1} \oplus 2^{n} A_{2} \oplus R_{R},
$$

whence $2^{n} B_{2} \lesssim 2^{n} A_{2} \oplus R_{R}$. Applying Lemma 2.7 a second time, we obtain decompositions $B_{2}=B_{3} \oplus B_{4}$ and $A_{2}=A_{3} \oplus A_{4}$ such that $B_{3} \cong A_{3}$ and $2^{n} B_{4} \lesssim R_{R}$. 
Now $B_{4} \lesssim 2^{n} B_{4} \lesssim R_{R}$, so $B_{4} \cong y R$ for some $y \in R$. Then as $2^{n}(y R) \lesssim R_{R}$, we obtain $N^{*}(y) \leqslant 1 / 2^{n}$ from Lemma 1.2. Similarly, since $2^{n} A_{4} \lesssim 2^{n} A_{2} \lesssim R_{R}$, we have $A_{4} \cong x R$ for some $x \in R$ satisfying $N^{*}(x) \leqslant 1 / 2^{n}$. Finally,

$$
\begin{aligned}
& A=A_{1} \oplus A_{2}=A_{1} \oplus A_{3} \oplus A_{4} \cong A_{1} \oplus A_{3} \oplus x R, \\
& B=B_{1} \oplus B_{2}=B_{1} \oplus B_{3} \oplus B_{4} \cong A_{1} \oplus A_{3} \oplus y R,
\end{aligned}
$$

hence we conclude that $v=[A]-[B]=[x R]-[y R]$.

LEMMA 2.10. Let $R$ be an $N^{*}$-complete regular ring, let $x_{1}, x_{2}, \ldots \in R$, and assume that $N^{*}\left(x_{n}\right)<1 / 2^{n}$ for all $n$. Then there exists $x \in R$ such that

$$
\left\|\left[x_{1} R\right]+\cdots+\left[x_{n} R\right]-[x R]\right\| \rightarrow 0
$$

as $n \rightarrow \infty$.

Proof. We may clearly assume that $R$ is nonzero.

For each $n$, Proposition 1.1 provides us with positive integers $s_{n}$ and $t_{n}$ such that $s_{n} / t_{n}<1 / 2^{n}$ and $t_{n}\left(x_{n} R\right) \lesssim s_{n} R_{R}$. Then since $2^{n} s_{n}=t_{n}$, we obtain $2^{n} s_{n}\left(x_{n} R\right) \lesssim$ $s_{n} R_{R}$, and so $2^{n}\left(x_{n} R\right) \lesssim R_{R}$, using Theorem 2.6. Cor ently,

$$
2^{n}\left(x_{1} R \oplus \cdots \oplus x_{n} R\right) \lesssim 2^{n-1} R_{R} \oplus 2^{n-2} R_{R} \oplus \cdots \oplus 2 R_{R} \oplus R_{R} \lesssim 2^{n} R_{R},
$$

whence $x_{1} R \oplus \cdots \oplus x_{n} R \lesssim R_{R}$ (Theorem 2.6 again).

As this holds for all $n$, we obtain $\oplus x_{n} R \lesssim R_{R}$, by [2, Proposition 4.8]. Thus $R$ contains an ascending sequence $A_{1} \leqslant A_{2} \leqslant \ldots$ of principal right ideals such that each

$$
A_{n} \cong x_{1} R \oplus \cdots \oplus x_{n} R
$$

By [2, Proposition 2.13], there exist orthogonal idempotents $e_{1}, e_{2}, \ldots$ in $R$ such that

$$
e_{1} R \oplus \cdots \oplus e_{n} R=A_{n}
$$

for all $n$. Note that $e_{n} R \cong A_{n} / A_{n-1} \cong x_{n} R$, when $N^{*}\left(e_{n}\right)=N^{*}\left(x_{n}\right)<1 / 2^{n}($ Lemma 1.2).

Now the partial sums of the series $\Sigma e_{n}$ are Cauchy with respect to $N^{*}$, hence there exists $e \in R$ such that $\sum e_{n} \rightarrow e$ in the $N^{*}$-metric. Note that $e$ is an idempotent, and $e_{n} e=e e_{n}=e_{n}$ for all $n$. For each $n$, we compute that

$$
N^{*}\left(e-e_{1}-\cdots-e_{n}\right)=N^{*}\left(\sum_{k=n+1}^{\infty} e_{k}\right) \leqslant \sum_{k=n+1}^{\infty} N^{*}\left(e_{k}\right)<\sum_{k=n+1}^{\infty} 1 / 2^{k}=1 / 2^{n},
$$

whence $2^{n}\left(\left(e-e_{1}-\cdots-e_{n}\right) R\right) \lesssim R_{R}$, using Proposition 1.1 and Theorem 2.6 again. Inasmuch as

$$
e R=e_{1} R \oplus \cdots \oplus e_{n} R \oplus\left(e-e_{1}-\cdots-e_{n}\right) R,
$$

it follows that $2^{n}(e R) \lesssim 2^{n}\left(e_{1} R \oplus \cdots \oplus e_{n} R\right) \oplus R_{R}$. Consequently,

$$
2^{n}\left(\left[e_{1} R\right]+\cdots+\left[e_{n} R\right]\right) \leqslant 2^{n}[e R] \leqslant 2^{n}\left(\left[e_{1} R\right]+\cdots+\left[e_{n} R\right]\right)+[R]
$$

in $K_{0}(R)$. Thus

$$
0 \leqslant 2^{n}\left([e R]-\left[e_{1} R\right]-\cdots-\left[e_{n} R\right]\right) \leqslant[R],
$$


from which we conclude that

$$
\left\|[e R]-\left[e_{1} R\right]-\cdots-\left[e_{n} R\right]\right\| \leqslant 1 / 2^{n} .
$$

Since each $\left[e_{n} R\right]=\left[x_{n} R\right]$, this proves that

$$
\left\|\left[x_{1} R\right]+\cdots+\left[x_{n} R\right]-[e R]\right\| \rightarrow 0,
$$

as desired.

THEOREM 2.11. If $R$ is an $N^{*}$-complete regular ring, then $\left(K_{0}(R),[R]\right)$ is an archimedean norm-complete interpolation group with order-unit.

Proof. Since $R$ is unit-regular (Theorem 2.3), it follows that $K_{0}(R)$ is a partially ordered (rather than just pre-ordered) abelian group [2, Proposition 15.2], and that $K_{0}(R)$ is an interpolation group [4, Proposition II.10.3]. Given $x, y \in K_{0}(R)$ such that $n x \leqslant y$ for all $n \in \mathbf{N}$, choose finitely generated projective right $R$-modules $A, B, C, D$ such that $x=[A]-[B]$ and $y=[C]-[D]$. Then

$$
[n A]-[n B]=n x \leqslant y \leqslant[C]
$$

and so $n A \lesssim n B \oplus C$, for all $n \in \mathbf{N}$. By Theorem $2.8, A \lesssim B$, whence $x \leqslant 0$. Therefore $K_{0}(R)$ is archimedean. In particular, it now follows from [4, Proposition I.6.2] that the pseudo-metric on $K_{0}(R)$ induced by $\|\cdot\|$ is actually a metric.

Finally, consider a Cauchy sequence $\left\{v_{1}, v_{2}, \ldots\right\}$ in $K_{0}(R)$. By passing to a subsequence, we may assume that $\left\|v_{n+1}-v_{n}\right\|<1 / 2^{n+1}$ for all $n$. Using Lemma 2.9, we obtain elements $x_{n}, y_{n} \in R$ such that

$$
v_{n+1}-v_{n}=\left[x_{n} R\right]-\left[y_{n} R\right] \text {, }
$$

while also $N^{*}\left(x_{n}\right) \leqslant 1 / 2^{n+1}$ and $N^{*}\left(y_{n}\right) \leqslant 1 / 2^{n+1}$. According to Lemma 2.10, there exist elements $x, y \in R$ such that

$$
\begin{aligned}
& \left\|\left[x_{1} R\right]+\cdots+\left[x_{n} R\right]-[x R]\right\| \rightarrow 0, \\
& \left\|\left[y_{1} R\right]+\cdots+\left[y_{n} R\right]-[y R]\right\| \rightarrow 0 .
\end{aligned}
$$

Since $v_{n+1}-v_{1}=\left[x_{1} R\right]+\cdots+\left[x_{n} R\right]-\left[y_{1} R\right]-\cdots-\left[y_{n} R\right]$ for all $n$, we conclude that

$$
\left\|\left(v_{n+1}-v_{1}\right)-([x R]-[y R])\right\| \rightarrow 0,
$$

and consequently $v_{n+1} \rightarrow v_{1}+[x R]-[y R]$. Therefore $\left(K_{0}(R),[R]\right)$ is normcomplete.

III. Archimedean norm-complete interpolation groups. Given an archimedean norm-complete interpolation group $(G, u)$ with order-unit, we study the state space $S(G, u)$, and the relationship between $G$ and the space of affine continuous realvalued functions on $S(G, u)$. In particular, we investigate extreme points and closed faces of $S(G, u)$, and relate them to ideals of $G$. These results will be applied, via $K_{0}$, to the ideal theory of $N^{*}$-complete regular rings.

Definition. Let $(G, u)$ be a partially ordered abelian group with order-unit. A state on $(G, u)$ is any positive homomorphism $s: G \rightarrow \mathbf{R}$ such that $s(u)=1$. The state space of $(G, u)$, denoted $S(G, u)$, is the set of all states on $(G, u)$. The state space is 
regarded as a subset of the linear topological space $\mathbf{R}^{G}$ (which is given the product topology), and as such is a compact convex set [2, Proposition 17.11]. If $G$ is an interpolation group, then $S(G, u)$ is a Choquet simplex [4, Theorem I.2.5].

Definition. The extreme boundary of a convex set $S$, denoted $\partial_{e} S$, is the set of all extreme points of $S$, that is, points $s \in S$ such that the only convex combinations $s=\alpha s^{\prime}+(1-\alpha) s^{\prime \prime}$ with $0 \leqslant \alpha \leqslant 1$ and $s^{\prime}, s^{\prime \prime} \in S$ are those for which $\alpha=0$, or $\alpha=1$, or $s^{\prime}=s^{\prime \prime}=s$. Now suppose that $S=S(G, u)$ for some partially ordered abelian group $(G, u)$ with order-unit. An extreme state $s$ in $\partial_{e} S$ is said to be discrete if $s(G)$ is a cyclic subgroup of $\mathbf{R}$. Note that if $s$ is discrete, then $s(G)=(1 / m) Z$ for some $m \in \mathbf{N}$, because $1=s(u) \in s(G)$. On the other hand, if $s$ is not discrete, then $s(G)$ is dense in $\mathbf{R}$.

Definition. We use $\operatorname{Aff}(S)$ to denote the partially ordered real Banach space of all affine continuous real-valued functions on $S$ (with the pointwise ordering and the supremum norm). Evaluation at elements of $G$ provides a map $\varphi: G \rightarrow \operatorname{Aff}(S)$, so that $\varphi(x)(s)=s(x)$ for all $x \in G$ and $s \in S$. Note that $\varphi$ is a positive homomorphism, and that $\varphi(u)$ is the constant function 1 . We refer to $\varphi$ as the natural map from $G$ to $\operatorname{Aff}(S)$. The map $\varphi$ is also norm-preserving; namely,

$$
\|\varphi(x)\|=\sup \{|s(x)|: s \in S\}=\|x\|
$$

for all $x \in G$ [4, Lemma I.6.1].

THEOREM 3.1. Let $(G, u)$ be an archimedean norm-complete interpolation group with order-unit, and set $S=S(G, u)$. For all discrete $s \in \partial_{e} S$, set $A_{s}=s(G)$; for all other $s \in \partial_{e} S$, set $A_{s}=\mathbf{R}$. Set

$$
A=\left\{p \in \operatorname{Aff}(S) \mid p(s) \in A_{s} \text { for all } s \in \partial_{e} S\right\} .
$$

Then the natural map from $G$ to $\operatorname{Aff}(S)$ provides an isomorphism of $(G, u)$ onto $(A, 1)$ (as partially ordered abelian groups with order-unit).

Proof. [3, Theorem 5.1].

COROLlARY 3.2. Let $(G, u)$ be an archimedean norm-complete interpolation group with order-unit. Then $G$ is lattice-ordered if and only if $\partial_{e} S(G, u)$ is compact.

Proof. [3, Corollary 5.4].

In order to apply Theorem 3.1 effectively, we must be able to identify $\partial_{e} S$ easily. Thus we develop criteria for deciding when states are extreme. For topological considerations, we also develop similar results for compact sets of extreme states, and for closed faces of $S$. We begin with a result relating the archimedean property to norm properties.

Proposition 3.3. Let $(G, u)$ be an interpolation group with order-unit. Then $G$ is archimedean if and only if $G$ is 2-unperforated and $G^{+}$is norm-closed in $G$.

Proof. If $G$ is archimedean, then $G$ is unperforated by [4, Lemma I.5.2]. Now consider elements $x_{1}, x_{2}, \ldots$ in $G^{+}$and $x \in G$ such that $x_{n} \rightarrow x$ in norm. We may assume that $\left\|x_{n}-x\right\|<1 / n$ for all $n$. According to [4, Proposition I.6.2], $n\left(x_{n}-x\right)$ $\leqslant u$, and consequently $n(-x) \leqslant u$. Since this holds for all $n \in \mathbf{N}$, the archimedean property implies that $-x \leqslant 0$, so that $x \in G^{+}$. Thus $G^{+}$is norm-closed in $G$. 
Conversely, assume that $G$ is 2-unperforated and that $G^{+}$is norm-closed in $G$. Given $a, b \in G$ such that $n a \leqslant b$ for all $n \in \mathbf{N}$, we must show that $a \leqslant 0$. Write $a=x-y$ for some $x, y \in G^{+}$, and choose $z \in G^{+}$with $b \leqslant z$. Then $n x \leqslant n y+z$ for all $n \in \mathbf{N}$, and we must show that $x \leqslant y$.

For all $n \in \mathbf{N}$, we have $2^{n} x \leqslant 2^{n} y+z$, hence [4, Lemma I.5.7] says that $x=v_{n}+$ $w_{n}$ for some $v_{n}, w_{n} \in G^{+}$such that $v_{n} \leqslant y$ and $2^{n} w_{n} \leqslant z$. Then $\left\|w_{n}\right\| \leqslant\|z\| / 2^{n}$, so that $w_{n} \rightarrow 0$, and consequently $v_{n} \rightarrow x$. Thus $y-v_{n} \rightarrow y-x$. Since each $y-v_{n}$ is in $G^{+}$, we conclude that $y-x$ is in $G^{+}$, as desired. Therefore $G$ is archimedean.

In particular, if $(G, u)$ is an archimedean interpolation group with order-unit, and we have norm-convergent sequences $x_{n} \rightarrow x$ and $y_{n} \rightarrow y$ in $G$ with $x_{n} \leqslant y_{n}$ for all $n$, then $x \leqslant y$.

THEOREM 3.4. Let $(G, u)$ be an interpolation group with order-unit, and let $s \in$ $S(G, u)$. Then $s$ is an extreme point of $S(G, u)$ if and only if

$$
\min \{s(x), s(y)\}=\sup \left\{s(z) \mid z \in G^{+} ; z \leqslant x ; z \leqslant y\right\}
$$

for all $x, y \in G^{+}$.

Proof. [3, Theorem 3.1].

Corollary 3.5. Let $(G, u)$ be an interpolation group with order-unit, and let $X$ be a compact subset of $\partial_{e} S(G, u)$. Given $x, y \in G^{+}$and a positive real number $\varepsilon$, there exists $z \in G^{+}$such that $z \leqslant x$ and $z \leqslant y$, while also

$$
s(z)>\min \{s(x), s(y)\}-\varepsilon
$$

for all $s \in X$.

Proof. Set $W=\left\{w \in G^{+} \mid w \leqslant x\right.$ and $\left.w \leqslant y\right\}$, and note, because of the interpolation property, that $W$ is upward directed. For each $w \in W$, set

$$
V(w)=\{s \in S(G, u) \mid s(w)>\min \{s(x), s(y)\}-\varepsilon\},
$$

which is an open subset of $S(G, u)$. In view of Theorem 3.4, we see that these $V(w)$ 's cover $\partial_{e} S(G, u)$, and so cover $X$. As $X$ is compact, it follows that

$$
X \subseteq V\left(w_{1}\right) \cup \cdots \cup V\left(w_{n}\right)
$$

for some $w_{1}, \ldots, w_{n} \in W$. Since $W$ is upward directed, there exists $z \in W$ such that all $w_{i} \leqslant z$, and $z$ has the desired properties.

COROLlARY 3.6. Let $(G, u)$ be an interpolation group with order-unit, let $a, b \in G^{+}$, and let $m \in \mathbf{N}$. Let $X$ be a compact subset of $\partial_{e} S(G, u)$, and assume that $s(m a) \leqslant s(b)$ for all $s \in X$. Given any positive real number $\varepsilon$, there exists $c \in G^{+}$such that $c \leqslant a$ and $m c \leqslant b$, while also $s(c)>s(a)-\varepsilon$ for all $s \in X$.

Proof. By Corollary 3.5, there exists $x \in G^{+}$such that $x \leqslant m a$ and $x \leqslant b$, while also $s(x)>s(m a)-\varepsilon$ for all $s \in X$. Then, using Riesz decomposition, $x=$ $x_{1}+\cdots+x_{m}$ for some $x_{i} \in G^{+}$satisfying $x_{i} \leqslant a$. For each $i$, note that $x-x_{i} \leqslant$ $(m-1) a$, whence $x-(m-1) a \leqslant x_{i}$. Since $0 \leqslant x_{i}$ for each $i$ as well, interpolation provides an element $c \in G$ such that

$$
x-(m-1) a \leqslant c \leqslant x_{i} \text { and } 0 \leqslant c \leqslant x_{i}
$$


for all $i$. Thus $c \in G^{+}$and $c \leqslant x_{1} \leqslant a$, while

$$
m c \leqslant x_{1}+\cdots+x_{m}=x \leqslant b .
$$

As $x-(m-1) a \leqslant c$, we have $a-c \leqslant m a-x$, whence

$$
s(a)-s(c) \leqslant s(m a)-s(x)<\varepsilon
$$

for all $s \in X$. Therefore $s(c)>s(a)-\varepsilon$ for all $s \in X$.

THEOREM 3.7. Let $(G, u)$ be an archimedean norm-complete interpolation group with order-unit, and let $X$ be a compact subset of $\partial_{e} S(G, u)$. Given any $x, y \in G$, there exist $z, w \in G$ such that $z \leqslant x \leqslant w$ and $z \leqslant y \leqslant w$, while also

$$
s(z)=\min \{s(x), s(y)\} \quad \text { and } \quad s(w)=\max \{s(x), s(y)\}
$$

for all $s \in X$. If $x, y \in G^{+}$, then such $z, w$ can be found in $G^{+}$.

Proof. First assume that $x, y \in G^{+}$. The rule $p(s)=\min \{s(x), s(y)\}$ defines a continuous map $p$ of $S(G, u)$ into $\mathbf{R}^{+}$. We construct elements $z_{1} \leqslant z_{2} \leqslant \ldots$ in $G^{+}$ such that each $z_{n} \leqslant x$ and $z_{n} \leqslant y$, while also

$$
s\left(z_{n}\right)>p(s)-\left(1 / 2^{n}\right) \quad \text { and } \quad\left\|z_{n+1}-z_{n}\right\| \leqslant 1 / 2^{n}
$$

for all $n$ and all $s \in X$. To begin, we obtain $z_{1}$ directly from Corollary 3.5.

Now assume that $z_{1}, \ldots, z_{n}$ have been constructed, for some $n$. According to Corollary 3.5, there exists $a \in G^{+}$such that $a \leqslant x$ and $a \leqslant y$, while also

$$
s(a)>p(s)-\left(1 / 2^{n+2}\right)
$$

for all $s \in X$. Since $z_{n} \leqslant x$ and $z_{n} \leqslant y$ as well, there is some $b \in G^{+}$satisfying $a \leqslant b \leqslant x$ and $z_{n} \leqslant b \leqslant y$. Note that

$$
s(b) \geqslant s(a)>p(s)-\left(1 / 2^{n+2}\right)
$$

for all $s \in X$.

The element $b-z_{n}$ lies in $G^{+}$, and for all $s \in X$ we have

$$
s\left(z_{n}\right)>\min \{s(x), s(y)\}-\left(1 / 2^{n}\right) \geqslant s(b)-\left(1 / 2^{n}\right),
$$

whence $s\left(2^{n}\left(b-z_{n}\right)\right)<1=s(u)$. By Corollary 3.6, there exists $c \in G^{+}$such that $c \leqslant b-z_{n}$ and $2^{n} c \leqslant u$, while also

$$
s(c)>s\left(b-z_{n}\right)-\left(1 / 2^{n+2}\right)
$$

for all $s \in X$. Set $z_{n+1}=z_{n}+c$, noting that $z_{n} \leqslant z_{n+1} \leqslant b \leqslant x$ and $z_{n+1} \leqslant b \leqslant y$. For all $s \in X$, we have

$$
s\left(z_{n+1}\right)=s\left(z_{n}\right)+s(c)>s(b)-\left(1 / 2^{n+2}\right)>p(s)-\left(1 / 2^{n+1}\right) .
$$

Since $0 \leqslant 2^{n}\left(z_{n+1}-z_{n}\right)=2^{n} c \leqslant u$, we also have $\left\|z_{n+1}-z_{n}\right\| \leqslant 1 / 2^{n}$, which completes the induction step.

Having constructed a Cauchy sequence $\left\{z_{1}, z_{2}, \ldots\right\}$ in $G$, we must have $z_{n} \rightarrow z$ for some $z \in G$. Since $0 \leqslant z_{n} \leqslant x$ and $0 \leqslant z_{n} \leqslant y$ for all $n$, we obtain $0 \leqslant z \leqslant x$ and $0 \leqslant z \leqslant y$. On the other hand, $z_{k} \geqslant z_{n}$ whenever $k \geqslant n$, hence $z \geqslant z_{n}$ for all $n$. Consequently, for any $s \in X$,

$$
\min \{s(x), s(y)\} \geqslant s(z) \geqslant s\left(z_{n}\right)>\min \{s(x), s(y)\}-\left(1 / 2^{n}\right),
$$

and thus $s(z)=\min \{s(x), s(y)\}$. 
Now consider arbitrary elements $x, y \in G$, and choose $a \in G^{+}$such that $x+a \geqslant$ 0 and $y+a \geqslant 0$. By the above, there exists $b \in G^{+}$such that $b \leqslant x+a$ and $b \leqslant y+a$, while also

$$
s(b)=\min \{s(x+a), s(y+a)\}
$$

for all $s \in X$. Setting $z=b-a$, we obtain $z \leqslant x$ and $z \leqslant y$, while

$$
s(z)=\min \{s(x), s(y)\}
$$

for all $s \in X$.

Finally, use the result above to obtain $c \in G$ such that $c \leqslant-x$ and $c \leqslant-y$, while also

$$
s(c)=\min \{s(-x), s(-y)\}
$$

for all $s \in X$. Set $w=-c$.

COROLlARY 3.8. If $(G, u)$ is an archimedean norm-complete interpolation group with order-unit, and $X$ is a compact subset of $\partial_{e} S(G, u)$, then $\operatorname{ker}(X)$ is an ideal of $G$, and $G / \operatorname{ker}(X)$ is a lattice-ordered abelian group.

Proof. Set $K=\operatorname{ker}(X)$. Clearly $K$ is a convex subgroup of $G$. Given $x \in K$, we have $s(x)=s(0)$ for all $s \in X$. According to Theorem 3.7, there exists $z \in G$ such that $z \leqslant x$ and $z \leqslant 0$, while also $s(z)=s(x)=s(0)$ for all $s \in X$. Thus $x-z$ and $-z$ are elements of $G^{+} \cap K$ satisfying $(x-z)-(-z)=x$, which proves that $K$ is a directed subgroup of $G$. Therefore $K$ is an ideal of $G$.

Now consider any elements $x+K$ and $y+K$ in $G / K$. According to Theorem 3.7, there exists $z \in G$ such that $z \leqslant x$ and $z \leqslant y$, while also $s(z)=\min \{s(x), s(y)\}$ for all $s \in X$. Then $z+K \leqslant x+K$ and $z+K \leqslant y+K$, and we claim that $z+K$ is the infimum of $x+K$ and $y+K$ in $G / K$.

Given $a+K$ in $G / K$ satisfying $a+K \leqslant x+K$ and $a+K \leqslant y+K$, we have $a \leqslant x+k^{\prime}$ and $a \leqslant y+k^{\prime \prime}$ for some $k^{\prime}, k^{\prime \prime} \in K$. Since $K$ is directed, we may choose $k \in K$ such that $k^{\prime} \leqslant k$ and $k^{\prime \prime} \leqslant k$, whence $a \leqslant x+k$ and $a \leqslant y+k$. Now

$$
a-k \leqslant x ; \quad a-k \leqslant y ; \quad z \leqslant x ; \quad z \leqslant y .
$$

Interpolating, we obtain $b \in G$ such that $a-k \leqslant b \leqslant x$ and $z \leqslant b \leqslant y$. In particular,

$$
s(z) \leqslant s(b) \leqslant \min \{s(x), s(y)\}=s(z)
$$

for all $s \in X$, whence $b-z \in K$. Thus

$$
a+K=(a-k)+K \leqslant b+K=z+K,
$$

proving that $z+K$ is indeed the infimum of $x+K$ and $y+K$.

Therefore $G / K$ is lattice-ordered.

We thank the referee for pointing out that the hypothesis of norm-completeness in Theorem 3.7 and Corollary 3.8 is essential, as the following example shows. (This is a simplified version of the referee's example.)

EXAMPLE 3.9. There exists an archimedean interpolation group $(G, u)$ with orderunit possessing an extreme state $s$ such that $\operatorname{ker}(s)=\{0\}$ but $G$ is not lattice-ordered. 
Proof. Let $G$ be the $\mathbf{Q}$-subspace of $\mathbf{R}^{2}$ spanned by the vectors $u=(1,1)$ and $v=(\pi,-\pi)$. Note that since $u$ and $v$ are $\mathbf{R}$-linearly independent, $G$ is dense in $\mathbf{R}^{2}$ in the usual Euclidean topology. Give $\mathbf{R}^{2}$ the direct product ordering, and give $G$ the relative ordering inherited from $\mathbf{R}^{2}$, so that $G^{+}=G \cap\left(\mathbf{R}^{2}\right)^{+}$. Then $\mathbf{R}^{2}$ and $G$ are archimedean partially ordered abelian groups, and $u$ is an order-unit in each of them.

To check interpolation, consider elements $x_{1}, x_{2}, y_{1}, y_{2}$ in $G$ satisfying $x_{i} \leqslant y_{j}$ for all $i, j$. Then each

$$
x_{i}=\left(a_{i}+b_{i} \pi, a_{i}-b_{i} \pi\right) \quad \text { and } \quad y_{j}=\left(c_{j}+d_{j} \pi, c_{j}-d_{j} \pi\right)
$$

for suitable $a_{i}, b_{i}, c_{j}, d_{j} \in \mathbf{Q}$. First assume that $a_{i}=c_{j}$ for some $i, j$, say $a_{1}=c_{1}$. Since

$$
a_{1}+b_{1} \pi \leqslant c_{1}+d_{1} \pi \quad \text { and } \quad a_{1}-b_{1} \pi \leqslant c_{1}-d_{1} \pi,
$$

it follows that $b_{1}=d_{1}$, whence $x_{1}=y_{1}$. In this case, $x_{i} \leqslant x_{1} \leqslant y_{j}$ for all $i, j$. Now assume that $a_{i} \neq c_{j}$ for all $i, j$. Then

$$
a_{i}+b_{i} \pi \neq c_{j}+d_{j} \pi \quad \text { and } \quad a_{i}-b_{i} \pi \neq c_{j}-d_{j} \pi
$$

for all $i, j$. Consequently, the set $W$ consisting of those $(\alpha, \beta)$ in $\mathbf{R}^{2}$ satisfying

$$
a_{i}+b_{i} \pi<\alpha<c_{j}+d_{j} \pi \quad \text { and } \quad a_{i}-b_{i} \pi<\beta<c_{j}-d_{j} \pi
$$

for all $i, j$ is a nonempty open subset of $\mathbf{R}^{2}$. Since $G$ is dense in $\mathbf{R}^{2}$, there exists an element $z$ in $G \cap W$, and $x_{i} \leqslant z \leqslant y_{j}$ for all $i, j$. Therefore $G$ is an interpolation group.

Define $t: \mathbf{R}^{2} \rightarrow \mathbf{R}$ by the rule $t(\alpha, \beta)=\alpha$, and note that $t$ is an extreme point of $S\left(\mathbf{R}^{2}, u\right)$. Let $s$ denote the restriction of $t$ to $G$. As $G^{+}=G \cap\left(\mathbf{R}^{2}\right)^{+}$, all states in $S(G, u)$ extend to states in $S\left(\mathbf{R}^{2}, u\right)$, by [2, Proposition 18.1]. Thus the restriction map $S\left(\mathbf{R}^{2}, u\right) \rightarrow S(G, u)$ is an affine homeomorphism, from which we see that $s$ is an extreme point of $S(G, u)$. It is clear that $\operatorname{ker}(s)=\{0\}$.

Consider any $x \in G$ satisfying $x \leqslant u$ and $x \leqslant v$. Then $x=(a+b \pi, a-b \pi)$ for some $a, b \in \mathbf{Q}$, and from the relations $x \leqslant u$ and $x \leqslant v$ we obtain $a \leqslant \alpha$, where

$$
\alpha=\min \{1-b \pi, 1+b \pi,(1-b) \pi,(b-1) \pi\} .
$$

Note that $\alpha$ must be irrational. Thus $a<\alpha$, and we may choose $c \in \mathbf{Q}$ such that $a<c<\alpha$. Setting $y=(c+b \pi, c-b \pi)$, we conclude that $x<y$ while also $y \leqslant u$ and $y \leqslant v$. Therefore the set $\{u, v\}$ has no infimum in $G$, proving that $G$ is not lattice-ordered.

In case the set $X$ in Corollary 3.8 is a singleton, we can precisely identify the quotient partially ordered abelian group $G / \operatorname{ker}(X)$, as follows.

THEOREM 3.10. Let $(G, u)$ be an archimedean norm-complete interpolation group with order-unit, and let $s \in \partial_{e} S(G, u)$. Then $\operatorname{ker}(s)$ is an ideal of $G$, and $s$ induces an isomorphism of $G / \operatorname{ker}(s)$ onto $s(G)$ as partially ordered abelian groups. Moreover, either $s(G)=\mathbf{R}$ or $s(G)=(1 / m) \mathbf{Z}$ for some $m \in \mathbf{N}$.

Proof. The subgroup $K=\operatorname{ker}(s)$ is an ideal of $G$ by Corollary 3.8. Obviously the induced map $\bar{s}: G / K \rightarrow s(G)$ is a group isomorphism, and a positive map as well. Given an element $x+K$ in $G / K$ with $\bar{s}(x+K) \geqslant 0$, we have $s(x) \geqslant 0=s(0)$. Then 
Theorem 3.7 provides us with an element $z \in G$ such that $z \leqslant x$ and $z \leqslant 0$, while also $s(z)=0$. Now $z \in K$ and $x-z \geqslant 0$, hence

$$
x+K=(x-z)+K \geqslant 0
$$

as well. Thus for all $a \in G / K$, we have $a \geqslant 0$ if and only if $\bar{s}(a) \geqslant 0$. Therefore $\bar{s}$ is an isomorphism of partially ordered abelian groups.

If $s$ is discrete, then $s(G)=(1 / m) \mathbf{Z}$ for some $m \in \mathbf{N}$. Now assume that $s$ is not discrete, so that $s(G)$ is dense in $\mathbf{R}$.

Given $\alpha \in \mathbf{R}$, we construct elements $x_{1}, x_{2}, \ldots$ in $G$ such that

$$
\alpha-\left(1 / 2^{n}\right)<s\left(x_{n}\right)<\alpha \quad \text { and } \quad\left\|x_{n+1}-x_{n}\right\| \leqslant 1 / 2^{n}
$$

for all $n$. To begin, we obtain $x_{1}$ from the density of $s(G)$ in $\mathbf{R}$.

Now assume that $x_{1}, \ldots, x_{n}$ have been constructed, for some $n$. Choose an element $a \in G$ such that

$$
\alpha-\left(1 / 2^{n+2}\right)<s(a)<\alpha .
$$

By Theorem 3.7, there exists $b \in G$ such that $b \geqslant x_{n}$ and $b \geqslant a$, while also $s(b)=$ $\max \left\{s\left(x_{n}\right), s(a)\right\}$. Note that

$$
\alpha-\left(1 / 2^{n+2}\right)<s(b)<\alpha .
$$

Since $s(b)<\alpha$ and $s\left(x_{n}\right)>\alpha-\left(1 / 2^{n}\right)$, we find that

$$
s\left(2^{n} b\right)<2^{n} \alpha<s\left(2^{n} x_{n}\right)+1,
$$

whence $s\left(2^{n}\left(b-x_{n}\right)\right)<1=s(u)$. As $b-x_{n} \in G^{+}$, Corollary 3.6 provides us with an element $c \in G^{+}$such that $c \leqslant b-x_{n}$ and $2^{n} c \leqslant u$, while also

$$
s(c)>s\left(b-x_{n}\right)-\left(1 / 2^{n+2}\right) \text {. }
$$

Set $x_{n+1}=x_{n}+c$, so that $x_{n} \leqslant x_{n+1} \leqslant b$. Thus $s\left(x_{n+1}\right) \leqslant s(b)<\alpha$, and

$$
s\left(x_{n+1}\right)=s\left(x_{n}\right)+s(c)>s(b)-\left(1 / 2^{n+2}\right)>\alpha-\left(1 / 2^{n+1}\right) .
$$

In addition, $\left\|x_{n+1}-x_{n}\right\|=\|c\| \leqslant 1 / 2^{n}$, which completes the induction step.

Now there exists $x \in G$ such that $x_{n} \rightarrow x$ in norm. Since

$$
\left|s\left(x_{n}\right)-s(x)\right|=\left|s\left(x_{n}-x\right)\right| \leqslant\left\|x_{n}-x\right\|
$$

for all $n$ [4, Lemma I.6.1], it follows that $s\left(x_{n}\right) \rightarrow s(x)$, and thus $s(x)=\alpha$. Therefore $s(G)=\mathbf{R}$, as desired.

Definition. A face of a convex set $S$ is a convex subset $F \subseteq S$ (possibly empty) such that whenever $s=\alpha s^{\prime}+(1-\alpha) s^{\prime \prime}$ is a positive convex combination with $s \in F$ and $s^{\prime}, s^{\prime \prime} \in S$, then $s^{\prime}, s^{\prime \prime} \in F$.

LEMMA 3.11. Let $(G, u)$ be an interpolation group with order-unit, and let $X$ be either a compact subset of $\partial_{e} S(G, u)$ or a closed face of $S(G, u)$. Let $t \in \partial_{e} S(G, u)$ such that $t \notin X$. Then there exists $x \in G^{+}$such that $t(x)>1$ but $s(x)<1$ for all $s \in X$.

Proof. Set $A=\left\{a \in G^{+} \mid t(a)>1\right\}$, and note that $2 u \in A$. Also, $A$ is downward directed, by Theorem 3.4. For all $a \in A$, set

$$
W(a)=\{s \in S(G, u) \mid s(a)<1\},
$$

which is an open subset of $S(G, u)$. We claim that these $W(a)$ 's cover $X$. 
Thus consider any $s \in X$. Since $\{t\}$ is a face of $S(G, u)$, and either $\{s\}$ or $X$ is a face of $S(G, u)$, we see that $s$ and $t$ lie in disjoint faces of $S(G, u)$. By [3, Lemma 2.8], $2 u=a+b$ for some $a, b \in G^{+}$such that $s(a)+t(b)<1$. Then $t(a)=2-t(b)>$ 1, whence $a \in A$ and $s \in W(a)$. Therefore the $W(a)$ 's do cover $X$, as claimed.

By compactness, $X \subseteq W\left(a_{1}\right) \cup \cdots \cup W\left(a_{n}\right)$ for some elements $a_{i} \in A$. As $A$ is downward directed, there exists $x \in A$ such that each $a_{i} \geqslant x$, and $x$ has the desired properties.

THEOREM 3.12. Let $(G, u)$ be an archimedean norm-complete interpolation group with order-unit, and let $X$ be a compact subset of $\partial_{e} S(G, u)$. Then

$$
X=\left\{s \in \partial_{e} S(G, u) \mid G^{+} \cap \operatorname{ker}(X) \subseteq \operatorname{ker}(s)\right\} .
$$

Proof. Consider $t \in \partial_{e} S(G, u)$ such that $t \notin X$. By Lemma 3.11, there exists $x \in G^{+}$such that $t(x)>1$ but $s(x)<1$ for all $s \in X$. Applying Theorem 3.7 to the elements $x, u \in G^{+}$and the compact subset $X \cup\{t\}$ of $\partial_{e} S(G, u)$, we obtain $y \in G^{+}$such that $y \geqslant x$ and $y \geqslant u$, while $t(y)>1$ and $s(y)=1$ for all $s \in X$. Consequently, $y-u$ is an element of $G^{+} \cap \operatorname{ker}(X)$ which does not lie in $\operatorname{ker}(t)$.

We now turn to closed faces of state spaces. The results above, concerning compact sets of extreme states, carry over fairly directly, with similar proofs.

THEOREM 3.13. Let $(G, u)$ be an interpolation group with order-unit, let $x, y \in G^{+}$, and let $F$ be a closed face of $S(G, u)$. Assume that $s(x) \leqslant s(y)$ for all $s \in F$. Given any positive real number $\varepsilon$, there exists $z \in G^{+}$such that $z \leqslant x$ and $z \leqslant y$, while also $s(z)>s(x)-\varepsilon$ for all $s \in F$.

Proof. [3, Theorem 3.4].

Corollary 3.14. Let $(G, u)$ be an interpolation group with order-unit, and let $F$ be a closed face of $S(G, u)$. Let $a, b \in G^{+}$and $m \in \mathbf{N}$, and assume that $s(m a) \leqslant s(b)$ for all $s \in F$. Given any positive real number $\varepsilon$, there exists $c \in G^{+}$such that $c \leqslant a$ and $m c \leqslant b$, while also $s(c)>s(a)-\varepsilon$ for all $s \in F$.

Proof. As Corollary 3.6, using Theorem 3.13 in place of Corollary 3.5.

THEOREM 3.15. Let $(G, u)$ be an archimedean norm-complete interpolation group with order-unit, let $x, y \in G$, and let $F$ be a closed face of $S(G, u)$. If $s(x) \leqslant s(y)$ for all $s \in F$, then there exist $z, w \in G$ such that $z \leqslant x \leqslant w$ and $z \leqslant y \leqslant w$, while also $s(z)=s(x)$ and $s(w)=s(y)$ for all $s \in F$. If $x, y \in G^{+}$, then such $z, w$ can be found in $G^{+}$.

Proof. As Theorem 3.7, using Theorem 3.13 and Corollary 3.14 in place of Corollaries 3.5 and 3.6.

COROLlaRY 3.16. If $(G, u)$ is an archimedean norm-complete interpolation group with order-unit, and $F$ is a closed face of $S(G, u)$, then $\operatorname{ker}(F)$ is an ideal of $G$.

Proof. As Corollary 3.8, using Theorem 3.15 in place of Theorem 3.7. 
THEOREM 3.17. Let $(G, u)$ be an archimedean norm-complete interpolation group with order-unit, and let $F$ be a closed face of $S(G, u)$. Then

$$
F=\left\{s \in S(G, u) \mid G^{+} \cap \operatorname{ker}(F) \subseteq \operatorname{ker}(s)\right\} .
$$

Proof. Set $F^{\prime}=\left\{s \in S(G, u) \mid G^{+} \cap \operatorname{ker}(F) \subseteq \operatorname{ker}(s)\right\}$. Clearly $F^{\prime}$ is a closed convex subset of $S(G, u)$, and we claim that $F^{\prime}$ is a face of $S(G, u)$ as well. Thus consider any positive convex combination $s=\alpha s^{\prime}+(1-\alpha) s^{\prime \prime}$ in $S(G, u)$ with $s \in F^{\prime}$. For any $x \in G^{+} \cap \operatorname{ker}(F)$, we have

$$
\alpha s^{\prime}(x)+(1-\alpha) s^{\prime \prime}(x)=0 ; \quad s^{\prime}(x) \geqslant 0 ; \quad s^{\prime \prime}(x) \geqslant 0
$$

and so $s^{\prime}(x)=s^{\prime \prime}(x)=0$. Therefore $s^{\prime}, s^{\prime \prime} \in F^{\prime}$, proving that $F^{\prime}$ is indeed a face of $S(G, u)$.

Being a compact convex set, $F^{\prime}$ equals the closure of the convex hull of its extreme boundary $\partial_{e} F^{\prime}$, by the Krein-Milman Theorem. Thus if $F^{\prime} \nsubseteq F$, there must be some $t \in \partial_{e} F^{\prime}$ which does not lie in $F$. As $F^{\prime}$ is a face of $S(G, u)$, we see that actually $t$ is an extreme point of $S(G, u)$.

By Lemma 3.11, there exists $x \in G^{+}$such that $t(x)>1$ but $s(x)<1$ for all $s \in F$. According to Theorem 3.15, there exists $w \in G^{+}$such that $x \leqslant w$ and $u \leqslant w$, while also $s(w)=1$ for all $s \in F$. Thus $w-u$ is an element of $G^{+} \cap \operatorname{ker}(F)$. On the other hand, $t(w) \geqslant t(x)>1$ and so $w-u$ is not in $\operatorname{ker}(t)$, which contradicts the fact that $t \in F^{\prime}$.

Therefore $F^{\prime} \subseteq F$. The reverse inclusion is automatic, hence $F=F^{\prime}$, as desired.

IV. $N^{*}$-complete regular rings. We apply the results of $\S I I$, via $K_{0}$, to the structure of $N^{*}$-complete regular rings $R$. The thrust of most of these results is that $R$ behaves much like a ring of sections of a sheaf of simple self-injective rings. Namely, for every maximal two-sided ideal $M$ of $R$, the factor ring $R / M$ is right and left self-injective, and many properties of $R$ and its projective modules are determined by what happens modulo these maximal two-sided ideals. For instance, $R$ is an $n \times n$ matrix ring (for some fixed $n \in \mathbf{N}$ ) if and only if each $R / M$ is an $n \times n$ matrix ring. For another example, given finitely generated projective right $R$-modules $A$ and $B$, we have $A \cong B$ if and only if $A / A M \cong B / B M$ for all $M$. Many of our results generalize parallel results for $\aleph_{0}$-continuous regular rings in $[4,7,8]$. At the end of the section, we indicate how our results relate to these papers.

All the results of this section depend on Theorems 2.3 and 2.11: That any $N^{*}$-complete regular ring $R$ is unit-regular, and that for such rings $\left(K_{0}(R),[R]\right)$ is an archimedean norm-complete interpolation group with order-unit. We shall use these results repeatedly without further reference to them. One other basic result is needed, to identify the state space of $\left(K_{0}(R),[R]\right)$.

Proposition 4.1. For any regular ring $R$, there is a natural affine homeomorphism $\theta: S\left(K_{0}(R),[R]\right) \rightarrow \mathbf{P}(R)$ such that $\theta(s)(x)=s([x R])$ for all $s$ in $S\left(K_{0}(R),[R]\right)$ and all $x \in R$.

Proof. [2, Proposition 17.12]. 
THEOREM 4.2. Let $R$ be an $N^{*}$-complete regular ring.

(a) If $X$ is a compact subset of $\partial_{e} P(R)$, then

$$
X=\left\{P \in \partial_{e} \mathbf{P}(R) \mid \operatorname{ker}(X) \subseteq \operatorname{ker}(P)\right\} .
$$

(b) If $F$ is a closed face of $\mathbf{P}(R)$, then

$$
F=\{P \in \mathbf{P}(R) \mid \operatorname{ker}(F) \subseteq \operatorname{ker}(P)\} .
$$

Proof. Set $S=S\left(K_{0}(R),[R]\right)$, and let $\theta: S \rightarrow \mathbf{P}(R)$ be the affine homeomorphism given in Proposition 4.1.

(a) Set $Y=\theta^{-1}(X)$, which is a compact subset of $\partial_{e} S$. Let $Q \in \partial_{e} \mathbf{P}(R)$ such that $\operatorname{ker}(X) \subseteq \operatorname{ker}(Q)$, and set $t=\theta^{-1}(Q)$. We shall show that $K_{0}(R)^{+} \cap \operatorname{ker}(Y)$ is contained in $\operatorname{ker}(t)$.

Given $a \in K_{0}(R)^{+} \cap \operatorname{ker}(Y)$, we have $a=[A]$ for some finitely generated projective right $R$-module $A$. Then

$$
A \cong x_{1} R \oplus \cdots \oplus x_{n} R \quad \text { and } \quad[A]=\left[x_{1} R\right]+\cdots+\left[x_{n} R\right]
$$

for some elements $x_{i} \in R$. For each $i$, we have $0 \leqslant\left[x_{i} R\right] \leqslant[A]$, whence $s\left(\left[x_{i} R\right]\right)=0$ for all $s \in Y$, and so $P\left(x_{i}\right)=0$ for all $P \in X$. Then each $x_{i}$ lies in $\operatorname{ker}(X)$, hence $x_{i} \in \operatorname{ker}(Q)$, and so $t\left(\left[x_{i} R\right]\right)=0$. Consequently,

$$
t(a)=t\left(\left[x_{1} R\right]\right)+\cdots+t\left(\left[x_{n} R\right]\right)=0 .
$$

Therefore $K_{0}(R)^{+} \cap \operatorname{ker}(Y) \subseteq \operatorname{ker}(t)$, as claimed.

Now Theorem 3.12 shows that $t \in Y$, and therefore $Q \in X$.

(b) As (a), using Theorem 3.17 in place of Theorem 3.12.

COROLLARY 4.3. If $M$ is a maximal two-sided ideal in an $N^{*}$-complete regular ring $R$, then $R / M$ is a simple, unit-regular, right and left self-injective ring. There is $a$ unique rank function on $R / M$, and $R / M$ is complete in the rank-metric.

Proof. According to Corollary $1.14, R / M$ is $N^{*}$-complete, hence there is no loss of generality in assuming that $M=0$.

Since $R$ is a nonzero unit-regular ring, [2, Corollary 18.5] shows that $\mathbf{P}(R)$ is nonempty. By the Krein-Milman Theorem, there exists at least one $P$ in $\partial_{e} \mathbf{P}(R)$. Note that $\operatorname{ker}(P)=0$, because $R$ is simple. Inasmuch as $\{P\}$ is a closed face of $\mathbf{P}(R)$, we now conclude from Theorem 4.2(b) that $\mathbf{P}(R)=\{P\}$. Thus $P$ is the unique rank function on $R$.

Now $N^{*}=P$, hence the $N^{*}$-metric on $R$ coincides with the $P$-metric. Therefore $R$ is complete in the $P$-metric. According to [2, Theorem 19.7], $R$ is thus right and left self-injective.

COROLlaRY 4.4. Let $R$ be an $N^{*}$-complete regular ring, and let $P \in \mathbf{P}(R)$. Then the following conditions are equivalent.

(a) $P$ is an extreme point of $\mathbf{P}(R)$.

(b) $\operatorname{ker}(P)$ is a maximal two-sided ideal of $R$.

(c) There is a unique pseudo-rank function on $R / \operatorname{ker}(P)$. 
Proof. Let $\bar{P}$ denote the rank function on $R / \operatorname{ker}(P)$ induced by $P$.

(a) $\Rightarrow(\mathrm{c})$ : Since $\{P\}$ is a closed face of $P(R)$, Theorem 4.2(b) says that $P$ is the only pseudo-rank function on $R$ whose kernel contains $\operatorname{ker}(P)$. Thus $\bar{P}$ is the only pseudo-rank function on $R / \operatorname{ker}(P)$.

(c) $\Rightarrow(\mathrm{b})$ : Since $R / \operatorname{ker}(P)$ is a unit-regular ring possessing a unique rank function, [2, Corollary 18.6] shows that $R / \operatorname{ker}(P)$ is a simple ring.

(b) $\Rightarrow$ (a): By Corollary $4.3, \bar{P}$ is the only rank function on $R / \operatorname{ker}(P)$, hence $P$ is the only pseudo-rank function on $R$ whose kernel contains $\operatorname{ker}(P)$. Given a positive convex combination $P=\alpha P_{1}+(1-\alpha) P_{2}$ in $\mathbf{P}(R)$, we see that $\operatorname{ker}(P) \subseteq \operatorname{ker}\left(P_{1}\right)$ because $\alpha>0$, whence $P_{1}=P$, and similarly $P_{2}=P$. Therefore $P$ is an extreme point of $\mathbf{P}(R)$.

With the help of Corollaries 4.3 and 4.4 , we can show that in any $N^{*}$-complete regular ring $R$, there is a natural bijection between $\partial_{e} \mathbf{P}(R)$ and the set of maximal two-sided ideals of $R$. In fact, this bijection is continuous with respect to the usual topology on the maximal ideal space, as follows.

Definition. For any ring $R$, we use $\operatorname{Max} \operatorname{Spec}(R)$ to denote the family of all maximal two-sided ideals of $R$, equipped with the usual hull-kernel topology.

THEOREM 4.5. Let $R$ be an $N^{*}$-complete regular ring.

(a) There is a continuous bijection $\theta: \partial_{e} \mathbf{P}(R) \rightarrow \operatorname{Max} \operatorname{Spec}(R)$ given by the rule $\theta(P)=\operatorname{ker}(P)$.

(b) $\theta$ maps compact subsets of $\partial_{e} \mathbf{P}(R)$ onto closed subsets of $\operatorname{Max} \operatorname{Spec}(R)$.

(c) $\theta$ is a homeomorphism if and only if $\partial_{e} \mathbf{P}(R)$ is compact, if and only if $\operatorname{Max} \operatorname{Spec}(R)$ is Hausdorff.

Proof. (a) It is clear from Corollaries 4.3 and 4.4 that $\theta$ defines a bijection of $\partial_{e} \mathbf{P}(R)$ onto $\operatorname{MaxSpec}(R)$. If $X$ is a closed subset of $\operatorname{MaxSpec}(R)$, then

$$
X=\{M \in \operatorname{MaxSpec}(R) \mid Y \subseteq M\}
$$

for some $Y \subseteq R$. Consequently,

$$
\theta^{-1}(X)=\left\{P \in \partial_{e} \mathbf{P}(R) \mid P(y)=0 \text { for all } y \in Y\right\},
$$

which is closed in $\partial_{e} \mathbf{P}(R)$. Therefore $\theta$ is continuous.

(b) If $X$ is a compact subset of $\partial_{e} \mathbf{P}(R)$, then

$$
X=\left\{P \in \partial_{e} \mathbf{P}(R) \mid \operatorname{ker}(X) \subseteq \theta(P)\right\},
$$

by Theorem 4.2(a). As a result,

$$
\theta(X)=\{M \in \operatorname{Max} \operatorname{Spec}(R) \mid \operatorname{ker}(X) \subseteq M\},
$$

which is closed in $\operatorname{MaxSpec}(R)$.

(c) Note that $\partial_{e} \mathbf{P}(R)$ is Hausdorff while $\operatorname{MaxSpec}(R)$ is compact. Thus if $\theta$ is a homeomorphism, then $\partial_{e} \mathbf{P}(R)$ must be compact and $\operatorname{Max} \operatorname{Spec}(R)$ must be Hausdorff. On the other hand, if $\partial_{e} \mathbf{P}(R)$ is compact, then we see from (b) that $\theta$ is a closed map, whence $\theta$ is a homeomorphism.

Now assume that $\operatorname{Max} \operatorname{Spec}(R)$ is Hausdorff. We shall show that $\partial_{e} \mathbf{P}(R)$ is compact, by showing that $\partial_{e} \mathbf{P}(R)$ is closed in $\mathbf{P}(R)$. 
If not, then some $P \in \mathbf{P}(R)$ lies in the closure of $\partial_{e} \mathbf{P}(R)$ but not in $\partial_{e} \mathbf{P}(R)$. Set $K=\operatorname{ker}(P)$, and note from Corollary 4.4 that there exist more than one pseudo-rank functions on $R / K$. Because of the Krein-Milman Theorem, there must exist at least two extreme points in $\mathrm{P}(R / K)$. As $R / K$ is $N^{*}$-complete (Theorem 1.13), it follows from (a) that $R / K$ has at least two maximal two-sided ideals. Thus there exist distinct $M_{1}$ and $M_{2}$ in $\operatorname{MaxSpec}(R)$ which contain $K$.

Since $\operatorname{Max} \operatorname{Spec}(R)$ is Hausdorff, there exist disjoint open sets $V_{1}$ and $V_{2}$ in $\operatorname{MaxSpec}(R)$ such that each $M_{i} \in V_{i}$. There are subsets $X_{i} \subseteq R$ such that each

$$
V_{i}=\left\{M \in \operatorname{MaxSpec}(R) \mid X_{i} \nsubseteq M\right\} .
$$

For each $i$, choose $x_{i} \in X_{i}$ such that $x_{i} \notin M_{i}$. Note that if $M$ is in $\operatorname{MaxSpec}(R)$ and $x_{i} \notin M$, then $M \in V_{i}$. Thus for any $M$ in $\operatorname{MaxSpec}(R)$, we must have either $x_{1} \in M$ or $x_{2} \in M$.

Inasmuch as $x_{i} \notin M_{i}$, we have $x_{i} \notin K$. Set

$$
W=\left\{Q \in \mathbf{P}(R) \mid Q\left(x_{1}\right)>0 \text { and } Q\left(x_{2}\right)>0\right\},
$$

which is an open subset of $\mathbf{P}(R)$. Note that $P \in W$, because each $x_{i} \notin \operatorname{ker}(P)$. Since $P$ lies in the closure of $\partial_{e} \mathbf{P}(R)$, there exists $Q$ in $W \cap \partial_{e} \mathbf{P}(R)$. But then $\operatorname{ker}(Q)$ is a maximal two-sided ideal of $R$ which contains neither $x_{i}$, a contradiction.

Thus $\partial_{e} \mathbf{P}(R)$ is indeed closed in $\mathbf{P}(R)$, and so is compact. Therefore $\theta$ is a homeomorphism in this case also.

COROLlARY 4.6. If $R$ is an $N^{*}$-complete regular ring, then the intersection of the maximal two-sided ideals of $R$ is zero. Consequently, $R$ is a subdirect product of simple, unit-regular, right and left self-injective rings.

Proof. If an element $x \in R$ lies in all maximal two-sided ideals, then by Theorem 4.5, $P(x)=0$ for all $P$ in $\partial_{e} \mathbf{P}(R)$. As the convex hull of $\partial_{e} \mathbf{P}(R)$ is dense in $\mathbf{P}(R)$ (the Krein-Milman Theorem), it follows that $P(x)=0$ for all $P$ in $\mathbf{P}(R)$. Then $N^{*}(x)=0$, whence $x=0$.

The subdirect product statement now follows from Corollary 4.3.

COROLlaRY 4.7. Let $R$ be an $N^{*}$-complete regular ring, and let $J$ be a two-sided ideal of $R$. Then the following conditions are equivalent.

(a) $R / J$ is $N^{*}$-complete.

(b) $J$ is $N^{*}$-closed in $R$.

(c) $J=\operatorname{ker}(X)$ for some $X \subseteq \mathbf{P}(R)$.

(d) $J=\cap Y$ for some $Y \subseteq \operatorname{Max} \operatorname{Spec}(R)$.

Proof. As $R$ is unit-regular, properties (a), (b), and (c) are equivalent by Theorem 1.13. We obtain (a) $\Rightarrow$ (d) from Corollary 4.6, while it follows from Theorem $4.5(\mathrm{a})$ that $(\mathrm{d}) \Rightarrow(\mathrm{c})$.

COROllaRY 4.8. Let $R$ be an $N^{*}$-complete regular ring. Then $K_{0}(R)$ is a lattice-ordered abelian group if and only if $\partial_{e} \mathbf{P}(R)$ is compact, if and only if $\operatorname{Max} \operatorname{Spec}(R)$ is Hausdorff. 
Proof. By Corollary 3.2 and Proposition 4.1, $K_{0}(R)$ is lattice-ordered if and only if $\partial_{e} \mathbf{P}(R)$ is compact. Theorem 4.5 shows that $\partial_{e} \mathbf{P}(R)$ is compact if and only if $\operatorname{MaxSpec}(R)$ is Hausdorff.

An alternate view of Theorem 4.5(a) is that for any $N^{*}$-complete regular ring $R$, there is another topology on $\partial_{e} \mathbf{P}(R)$, coarser than the usual topology, with respect to which the bijection $P \mapsto \operatorname{ker}(P)$ provides a homeomorphism of $\partial_{e} \mathbf{P}(R)$ onto $\operatorname{Max} \operatorname{Spec}(R)$. This topology coincides with a known topology defined on extreme boundaries of compact convex sets, as follows.

Definition. Let $K$ be any compact convex set, and let $\mathscr{F}$ denote the family of subsets of $\partial_{e} K$ of the form $F \cap \partial_{e} K$, where $F$ is a closed split-face of $K$. (See [1, p. 133] for the definition of a split-face.) According to [1, Proposition II.6.20], $\mathscr{F}$ is closed under finite unions and arbitrary intersections. Thus $\mathscr{F}$ is the family of closed sets for a topology on $\partial_{e} K$, known as the facial topology [1, p. 143]. When $K$ is a Choquet simplex, every closed face of $K$ is a split-face [1, Theorem II.6.22], hence in this case $\mathcal{F}$ consists of all sets of the form $F \cap \partial_{e} K$ where $F$ is any closed face of $K$. This simplification will apply in our considerations because $\mathbf{P}(R)$, for any regular ring $R$, is a Choquet simplex [2, Theorem 17.5].

THEOREM 4.9. Let $R$ be an $N^{*}$-complete regular ring. If $\partial_{e} \mathbf{P}(R)$ is given the facial topology, then the rule $P \mapsto \operatorname{ker}(P)$ defines a homeomorphism of $\partial_{e} \mathbf{P}(R)$ onto $\operatorname{Max} \operatorname{Spec}(R)$.

Proof. By Theorem 4.5(a), the rule $\theta(P)=\operatorname{ker}(P)$ defines a bijection $\theta$ of $\partial_{e} \mathbf{P}(R)$ onto $\operatorname{Max} \operatorname{Spec}(R)$. If $X$ is a closed subset of $\operatorname{Max} \operatorname{Spec}(R)$, then

$$
X=\{M \in \operatorname{MaxSpec}(R) \mid Y \subseteq M\},
$$

for some $Y \subseteq R$. Set $F=\{P \in \mathbf{P}(R) \mid Y \subseteq \operatorname{ker}(P)\}$, and recall that $F$ is a closed face of $\mathbf{P}(R)$ [2, Lemma 16.18]. Observing that

$$
\theta^{-1}(X)=F \cap \partial_{e} \mathbf{P}(R),
$$

we see that $\theta^{-1}(X)$ is closed in $\partial_{e} \mathbf{P}(R)$ in the facial topology. Thus $\theta$ is continuous with respect to the facial topology.

If $F$ is any closed face of $P(R)$, then Theorem 4.2(b) says that

$$
F=\{P \in \mathbf{P}(R) \mid \operatorname{ker}(F) \subseteq \operatorname{ker}(P)\} .
$$

As a result,

$$
\boldsymbol{\theta}\left(F \cap \partial_{e} \mathbf{P}(R)\right)=\{M \in \operatorname{Max} \operatorname{Spec}(R) \mid \operatorname{ker}(F) \subseteq M\},
$$

which is closed in $\operatorname{MaxSpec}(R)$. Thus $\theta$ is a closed map with respect to the facial topology.

Part of Theorem 4.5(c) may be proved using Theorem 4.9, because of the general result that in a Choquet simplex $K$, the facial topology on $\partial_{e} K$ is Hausdorff if and only if the usual topology on $\partial_{e} K$ is compact [1, Theorem II.7.8].

We now turn to the study of finitely generated projective modules over an $N^{*}$-complete regular ring $R$. In particular, we show that their isomorphism classes are determined both by the values of pseudo-rank functions on $R$, and by their isomorphism classes modulo maximal two-sided ideals of $R$. 
THEOREM 4.10. Let $R$ be an $N^{*}$-complete regular ring, let $A$ and $B$ be finitely generated projective right $R$-modules, and get

$$
A \cong x_{1} R \oplus \cdots \oplus x_{n} R ; \quad B \cong y_{1} R \oplus \cdots \oplus y_{k} R
$$

for some elements $x_{1}, \ldots, x_{n}, y_{1}, \ldots, y_{k} \in R$.

(a) $A \lesssim B$ if and only if $A / A M \lesssim B / B M$ for all $M \in \operatorname{MaxSpec}(R)$, if and only if

$$
P\left(x_{1}\right)+\cdots+P\left(x_{n}\right) \leqslant P\left(y_{1}\right)+\cdots+P\left(y_{k}\right)
$$

for all $P \in \partial_{e} \mathbf{P}(R)$.

(b) $A \cong B$ if and only if $A / A M \cong B / B M$ for all $M \in \operatorname{MaxSpec}(R)$, if and only if

$$
P\left(x_{1}\right)+\cdots+P\left(x_{n}\right)=P\left(y_{1}\right)+\cdots+P\left(y_{k}\right)
$$

for all $P \in \partial_{e} \mathbf{P}(R)$.

Proof. (a) If $A \lesssim B$, then obviously $A / A M \lesssim B / B M$ for all $M$ in $\operatorname{MaxSpec}(R)$.

Now assume that $A / A M \lesssim B / B M$ for all $M \in \operatorname{MaxSpec}(R)$. Consider any $P$ in $\partial_{e} \mathbf{P}(R)$, and set $M=\operatorname{ker}(P)$, which is in $\operatorname{MaxSpec}(R)$ by Corollary 4.4. Let $\bar{P}$ denote the rank function induced on $R / M$ by $P$. Using $x \mapsto \bar{x}$ for the natural map $R \rightarrow R / M$, we have

$$
\bar{x}_{1}(R / M) \oplus \cdots \oplus \bar{x}_{n}(R / M) \cong A / A M \lesssim B / B M \cong \bar{y}_{1}(R / M) \oplus \cdots \oplus \bar{y}_{k}(R / M) .
$$

Applying [2, Proposition 16.1], we obtain

$$
\bar{P}\left(\bar{x}_{1}\right)+\cdots+\bar{P}\left(\bar{x}_{n}\right) \leqslant \bar{P}\left(\bar{y}_{1}\right)+\cdots+\bar{P}\left(\bar{y}_{k}\right),
$$

and consequently $\Sigma P\left(x_{i}\right) \leqslant \Sigma P\left(y_{j}\right)$.

Finally, assume that $\Sigma P\left(x_{i}\right) \leqslant \Sigma P\left(y_{j}\right)$ for all $P \in \partial_{e} \mathbf{P}(R)$. In view of Proposition 4.1, it follows that

$$
s([A])=s\left(\left[x_{1} R\right]+\cdots+\left[x_{n} R\right]\right) \leqslant s\left(\left[y_{1} R\right]+\cdots+\left[y_{k} R\right]\right)=s([B])
$$

for all extreme states $s$ on $\left(K_{0}(R),[R]\right)$. Since $K_{0}(R)$ is archimedean, we conclude from [4, Proposition I.5.3] that $[A] \leqslant[B]$. Therefore $A \lesssim B$.

(b) This follows directly from (a) and the unit-regularity of $R$.

Definition. Let $R$ be any regular ring, and set $S=S\left(K_{0}(R),[R]\right)$. By Proposition 4.1, there is a natural affine homeomorphism $\theta: S \rightarrow \mathbf{P}(R)$ such that $\theta(s)(x)=$ $s([x R])$ for all $s \in S$ and all $x \in R$. Then $\theta$ induces an isomorphism $\theta^{*}$ of $\operatorname{Aff}(\operatorname{P}(R))$ onto $\operatorname{Aff}(S)$, as partially ordered Banach spaces. We also have the natural evaluation map $\varphi$ from $K_{0}(R)$ to $\operatorname{Aff}(S)$. Composing $\varphi$ with $\left(\theta^{*}\right)^{-1}$, we obtain a positive homomorphism

$$
\psi=\left(\theta^{*}\right)^{-1} \varphi: K_{0}(R) \rightarrow \operatorname{Aff}(\mathbf{P}(R))
$$

such that $\psi(x)(P)=\theta^{-1}(P)(x)$ for all $x \in K_{0}(R)$ and all $P \in \mathbf{P}(R)$. In particular, $\psi([x R])(P)=P(x)$ for all $x \in R$ and all $P \in \mathbf{P}(R)$. We refer to $\psi$ as the natural map from $K_{0}(R)$ to $\operatorname{Aff}(\mathbf{P}(R))$. Note that $\psi([R])$ is the constant function 1 . 
THEOREM 4.11. Let $R$ be an $N^{*}$-complete regular ring. Whenever $P \in \partial_{e} \mathbf{P}(R)$ and $R / \operatorname{ker}(P)$ is isomorphic to an $m \times m$ matrix ring over a division ring, set $A_{P}=$ $(1 / m) \mathbf{Z}$; for all other $P \in \partial_{e} \mathbf{P}(R)$, set $A_{P}=\mathbf{R}$. Set

$$
A=\left\{q \in \operatorname{Aff}(\mathbf{P}(R)) \mid q(P) \in A_{P} \text { for all } P \in \partial_{e} \mathbf{P}(R)\right\} .
$$

Then the natural map $\psi: K_{0}(R) \rightarrow \operatorname{Aff}(\mathbf{P}(R))$ provides an isomorphism of $\left(K_{0}(R),[R]\right)$ onto $(A, 1)$ (as partially ordered abelian groups with order-unit).

Proof. Define $S, \theta, \varphi$ as in the definition above, so that $\psi=\left(\theta^{*}\right)^{-1} \varphi$. For all discrete $s \in \partial_{e} S$, set $B_{s}=s\left(K_{0}(R)\right)$; for all other $s \in \partial_{e} S$, set $B_{s}=\mathbf{R}$. Set

$$
B=\left\{p \in \operatorname{Aff}(S) \mid p(S) \in B_{s} \text { for all } s \in \partial_{e} S\right\} .
$$

According to Theorem 3.1, $\varphi$ provides an isomorphism of $\left(K_{0}(R),[R]\right)$ onto $(B, 1)$. Thus we need only show that $\left(\theta^{*}\right)^{-1}$ restricts to an isomorphism of $(B, 1)$ onto $(A, 1)$. To prove this, it suffices to show that $A_{\theta(s)}=B_{s}$ for all $s \in \partial_{e} S$.

Thus let $s \in \partial_{e} S$, and set $P=\theta(s)$ and $M=\operatorname{ker}(P)$, so that $P \in \partial_{e} \mathbf{P}(R)$ and $M$ is a maximal two-sided ideal of $R$. Let $\bar{P}$ be the rank function induced by $P$ on $R / M$.

If $s$ is discrete, then $s$ induces an isomorphism of $K_{0}(R) / \operatorname{ker}(s)$ onto $(1 / m) \mathbf{Z}$ for some $m \in \mathbf{N}$ (Theorem 3.10), whence

$$
\bar{P}(R / M)=P(R)=\{0,1 / m, 2 / m, \ldots, 1\} .
$$

Choosing $x \in R / M$ such that $\bar{P}(x)=1 / m$, we infer that $x(R / M)$ is a minimal right ideal of $R / M$, whence $R / M$ is a simple artinian ring. Thus $R / M \cong M_{k}(D)$ for some $k \in \mathbf{N}$ and some division ring $D$. There is a unique rank function on $M_{k}(D)$, and its range of values is $\{0,1 / k, 2 / k, \ldots, 1\}$ [2, Corollary 16.6]. Consequently, we must have $k=m$, and so

$$
A_{\theta(s)}=A_{P}=(1 / m) \mathbf{Z}=s\left(K_{0}(R)\right)=B_{s} .
$$

If $s$ is not discrete, then $s$ induces an isomorphism of $K_{0}(R) / \operatorname{ker}(s)$ onto $\mathbf{R}$, by Theorem 3.10, whence

$$
\bar{P}(R / M)=P(R)=[0,1] .
$$

In this case, $R / M$ cannot be a simple artinian ring, hence

$$
A_{\theta(s)}=A_{P}=\mathbf{R}=B_{s},
$$

as desired.

COROLlARY 4.12. Let $R$ be an $N^{*}$-complete regular ring. If $R$ has no simple artinian homomorphic images, then the natural map from $K_{0}(R)$ to $\operatorname{Aff}(\operatorname{P}(R))$ is an isomorphism of partially ordered abelian groups.

THEOREM 4.13. Let $R$ be an $N^{*}$-complete regular ring, let $B$ be a finitely generated projective right $R$-module, and let $n \in \mathbf{N}$. Assume, for each maximal two-sided ideal $M$ of $R$ such that $R / M$ is artinian, that $B / B M$ is a direct sum of $n$ pairwise isomorphic submodules. Then $B$ is a direct sum of $n$ pairwise isomorphic submodules. In particular, if $R$ has no simple artinian homomorphic images, then this holds for all $n \in \mathbf{N}$. 
Proof. In the notation of Theorem 4.11, we wish to show that the function $\psi([B]) / n$ lies in $A$. Thus consider any $P \in \partial_{e} \mathbf{P}(R)$ such that $R / \operatorname{ker}(P) \cong M_{m}(D)$ for some $m \in \mathbf{N}$ and some division ring $D$. The ideal $M=\operatorname{ker}(P)$ is a maximal two-sided ideal of $R$, and $R / M$ has a unique simple right module $S$. Then $B / B M \cong k S$ for some $k \in \mathbf{Z}^{+}$, and $\psi([B])(P)=k / m$. Since $B / B M$ is assumed to be a direct sum of $n$ pairwise isomorphic submodules, $n$ must divide $k$, hence

$$
\psi([B])(P) / n=(k / n) / m \in(1 / m) \mathbf{Z}=A_{P} .
$$

As this holds for all $P \in \partial_{e} \mathbf{P}(R)$ such that $R / \operatorname{ker}(P)$ is simple artinian, we find that $\psi([B]) / n$ does lie in $A$, as desired.

In fact, $\psi([B]) / n$ must lie in $A^{+}$. Because of the isomorphism given in Theorem 4.11, it follows that there exists $x \in K_{0}(R)^{+}$such that $n x=[B]$. Then $x=[C]$ for some finitely generated projective right $R$-module $C$, and $[n C]=[B]$. Therefore $B \cong n C$.

COROLlaRY 4.14. Let $R$ be an $N^{*}$-complete regular ring, and let $n \in \mathbf{N}$. If every simple artinian homomorphic image of $R$ is an $n \times n$ matrix ring, then $R$ is an $n \times n$ matrix ring. In particular, if $R$ has no simple artinian homomorphic images, then $R$ is an $n \times n$ matrix ring for every $n \in \mathbf{N}$.

As another application of the affine representation of $K_{0}$ of an $N^{*}$-complete regular ring (Theorem 4.11 and Corollary 4.12), we derive criteria for the following properties.

Definition. Let $R$ be a unit-regular ring. We say that $R$ satisfies countable interpolation provided that given any elements $x_{1}, x_{2}, \ldots$ and $y_{1}, y_{2}, \ldots$ in $R$ satisfying $x_{i} R \lesssim y_{j} R$ for all $i, j$, there exists $z \in R$ such that $x_{i} R \lesssim z R \lesssim y_{j} R$ for all $i, j$. According to [4, Proposition II.12.1], this property is left-right symmetric, and is equivalent to the countable interpolation property in $K_{0}(R)$. The ring $R$ is said to satisfy general comparability provided that given any $x, y \in R$, there exists a central idempotent $e \in R$ such that $e x R \lesssim e y R$ and $(1-e) y R \lesssim(1-e) x R$.

Definition. Let $X$ be a compact Hausdorff space. The space $X$ is said to be an $F$-space if disjoint open $F_{\sigma}$ subsets of $X$ always have disjoint closures. The space $X$ is said to be basically disconnected if the closure of every open $F_{\sigma}$ subset of $X$ is open.

THEOREM 4.15. Let $R$ be an $N^{*}$-complete regular ring with no simple artinian homomorphic images, and assume that $\partial_{e} \mathbf{P}(R)$ is compact. Then $R$ has countable interpolation if and only if $\partial_{e} \mathbf{P}(R)$ is an $F$-space, if and only if $\operatorname{Max} \operatorname{Spec}(R)$ is an F-space.

Proof. Set $X=\partial_{e} \mathbf{P}(R)$, and note from Theorem 4.5 that $X$ is homeomorphic to $\operatorname{Max} \operatorname{Spec}(R)$. Combining Corollary 4.12 with [1, Proposition II.3.13], we see that

$$
K_{0}(R) \cong \operatorname{Aff}(\mathbf{P}(R)) \cong C(X, \mathbf{R})
$$

as partially ordered abelian groups. Thus $R$ has countable interpolation if and only if $C(X, \mathbf{R})$ satisfies the countable interpolation property (as a partially ordered set). According to [9, Theorem 1.1], this happens if and only if $X$ is an $F$-space. 
Theorem 4.15 may fail if $R$ is allowed to have simple artinian homomorphic images, as the following example shows.

EXAMPLE 4.16. There exists an $N^{*}$-complete regular ring $R$ such that $\partial_{e} \mathbf{P}(R)$ is a compact $F$-space, but $R$ does not have countable interpolation.

Proof. Choose a field $K$, set $R_{n}=M_{2}(K)$ for all $n \in \mathbf{N}$, and set

$$
R=\left\{x \in \prod R_{n} \mid x_{n} \in K \text { for all but finitely many } n \in \mathbf{N}\right\} \text {. }
$$

Clearly $R$ is a regular ring whose index of nilpotence is 2 . By Theorem $1.3, R$ is $N^{*}$-complete.

The Boolean algebra $B(R)$ of central idempotents in $R$ is a direct product of copies of $\{0,1\}$ and so is complete. Consequently, its maximal ideal space $B S(R)$ is compact, Hausdorff, and extremally disconnected. In particular, $B S(R)$ is a compact $F$-space. Observing that $R$ satisfies general comparability, we see by [2, Theorem 16.28] that $\partial_{e} \mathbf{P}(R)$ is homeomorphic to $B S(R)$. Thus $\partial_{e} \mathbf{P}(R)$ is a compact $F$-space.

Choose $x_{1}, x_{2}, \ldots$ and $y_{1}, y_{2}, \ldots$ in $R$ so that $\operatorname{rank}\left(x_{k n}\right)=\operatorname{rank}\left(y_{k n}\right)=1$ for all $n=1, \ldots, k$, whereas $x_{k n}=0$ and $y_{k n}=1$ for all $n>k$. Clearly $x_{i} R \lesssim y_{j} R$ for all $i, j$. If there exists $z \in R$ such that $x_{i} R \lesssim z R \lesssim y_{j} R$ for all $i, j$, then $x_{n n} R \lesssim z_{n} R \lesssim$ $y_{n n} R$ for all $n \in \mathrm{N}$, whence $\operatorname{rank}\left(z_{n}\right)=1$ for all $n$. But then $z_{n} \notin K$ for all $n$, which is impossible for an element $z \in R$. Therefore $R$ does not satisfy countable interpolation.

THEOREM 4.17. Let $R$ be an $N^{*}$-complete regular ring. If $\partial_{e} \mathbf{P}(R)$ is a compact totally disconnected $F$-space, then $R$ satisfies general comparability.

Proof. Set $\Delta=\partial_{e} \mathbf{P}(R)$. Given $x, y \in R$, set

$$
X=\{P \in \Delta \mid P(x)<P(y)\} ; \quad Y=\{P \in \Delta \mid P(x)>P(y)\} .
$$

Inasmuch as the rule $P \mapsto P(x)-P(y)$ defines a continuous real-valued map on $\Delta$, we see that $X$ and $Y$ are disjoint open $F_{\sigma}$ subsets of $\Delta$. Since $\Delta$ is assumed to be an $F$-space, the closures of $X$ and $Y$ must be disjoint. Consequently, it follows from the total disconnectedness of $\Delta$ that there is a clopen set $V \subseteq \Delta$ such that $X \subseteq V$ and $Y \subseteq \Delta-V$.

Now let $\theta: \Delta \rightarrow \operatorname{MaxSpec}(R)$ be the homeomorphism given by Theorem 4.5, so that $\theta(V)$ is a clopen subset of $\operatorname{MaxSpec}(R)$. As the intersection of the maximal two-sided ideals of $R$ is zero (Corollary 4.6), there must exist a central idempotent $e \in R$ such that

$$
\theta(V)=\{M \in \operatorname{MaxSpec}(R) \mid e \notin M\} .
$$

Given any $P \in V$, we have $\operatorname{ker}(P) \in \theta(V)$ and so $e \notin \operatorname{ker}(P)$. Then $1-e$ lies in $\operatorname{ker}(P)$, hence $P(x)=P(e x)$ and $P(y)=P(e y)$, by [2, Lemma 16.2]. Consequently, $P(e x)<P(e y)$ for all $P \in X$, and $P(e x)=P(e y)$ for all $P \in V-X$. On the other hand, for $P \in \Delta-V$, we have $\operatorname{ker}(P) \notin \theta(V)$ and so $e \in \operatorname{ker}(P)$, whence $P(e x)=$ $0=P(e y)$. Thus $P(e x) \leqslant P(e y)$ for all $P \in \Delta$, hence $e x R \lesssim e y R$, by Theorem 4.10. Similarly, $(1-e) y R \lesssim(1-e) x R$. Therefore $R$ satisfies general comparability. 
The proof of Theorem 4.17 can be considerably shortened if $R$ has no simple artinian homomorphic images. For in this case $R$ has countable interpolation by Theorem 4.15, and then [4, Theorem II.14.7] shows that $R$ satisfies general comparability.

COROLlaRY 4.18. Let $R$ be an $N^{*}$-complete regular ring with no simple artinian homomorphic images. Then $R$ satisfies general comparability if and only if $\partial_{e} \mathbf{P}(R)$ is a compact totally disconnected $F$-space, if and only if $\operatorname{Max} \operatorname{Spec}(R)$ is a Hausdorff totally disconnected F-space.

Proof. It is clear from Theorem 4.5 that $\partial_{e} \mathbf{P}(R)$ is a compact totally disconnected $F$-space if and only if $\operatorname{Max} \operatorname{Spec}(R)$ is a Hausdorff totally disconnected $F$-space. These conditions imply general comparability in $R$ by Theorem 4.17 .

Conversely, assume that $R$ has general comparability. It follows from [2, Theorem 16.28] that $\partial_{e} \mathbf{P}(R)$ is compact and totally disconnected.

Now consider any disjoint open $F_{\sigma}$ subsets $X$ and $Y$ in $\partial_{e} \mathbf{P}(R)$. Then there exists a continuous real-valued function $f$ on $\partial_{e} \mathbf{P}(R)$ such that $f>0$ on $X$ and $f<0$ on $Y$. (This is an exercise in applying Urysohn's Lemma, which we leave to the reader.) As $\partial_{e} \mathbf{P}(R)$ is compact, we can modify $f$ to obtain a continuous function

$$
g: \partial_{e} \mathbf{P}(R) \rightarrow[0,1]
$$

such that $g>1 / 2$ on $X$ and $g<1 / 2$ on $Y$. By [1, Proposition II.3.13], $g$ extends to an affine continuous function $g^{*}: \mathbf{P}(R) \rightarrow[0,1]$, to which we apply Corollary 4.12. Since $0 \leqslant g^{*} \leqslant 1$, we obtain an element $b \in K_{0}(R)$ such that $0 \leqslant b \leqslant[R]$ and the map induced by $b$ in $\operatorname{Aff}(\mathbf{P}(R))$ coincides with $g^{*}$. Thus $b=[x R]$ for some $x \in R$, and $g^{*}(P)=P(x)$ for all $P \in \mathbf{P}(R)$.

We use general comparability to compare the projective modules $2(x R)$ and $R_{R}$, via [2, Proposition 8.8]. Thus we obtain a central idempotent $e \in R$ such that

$$
2(e x R) \lesssim e R \quad \text { and } \quad(1-e) R \lesssim 2((1-e) x R) .
$$

Consider any $P \in X$, so that $P(x)=g(P)>1 / 2$. If $e \notin \operatorname{ker}(P)$, then $1-e$ is in $\operatorname{ker}(P)$, because $\operatorname{ker}(P)$ is a maximal two-sided ideal of $R$ (Corollary 4.4). But then

$$
2 P(x)=2 P(e x) \leqslant P(e)=1
$$

(since $2($ exR $) \lesssim e R)$, which contradicts the fact that $P(x)>1 / 2$. Thus $e \in \operatorname{ker}(P)$ and so $P(e)=0$. Similarly, for any $P \in Y$ we have $1-e \in \operatorname{ker}(P)$ and so $P(e)=1$. As the map $P \mapsto P(e)$ is a continuous map from $P(R)$ to $\mathbf{R}$, we conclude that $X$ and $Y$ must have disjoint closures.

Therefore $\partial_{e} \mathbf{P}(R)$ is an $F$-space.

Corollary 4.18 may fail if $R$ is allowed to have simple artinian homomorphic images, as the following example shows.

EXAMPle 4.19. There exists an $N^{*}$-complete regular ring $R$ such that $R$ satisfies general comparability, but $\partial_{e} \mathbf{P}(R)$ is not an $F$-space.

Proof. Choose a field $K$, and let $R$ be the ring of all eventually constant sequences

$$
\left(\alpha_{1}, \alpha_{2}, \ldots, \alpha_{n}, \alpha, \alpha, \alpha, \ldots\right)
$$


of elements of $K$. Clearly $R$ is a regular ring whose index of nilpotence is 1 . By Theorem 1.3, $R$ is $N^{*}$-complete. It is also clear that $R$ satisfies general comparability.

The maximal ideals of $R$ are easily identified, from which one sees that $\operatorname{Max} \operatorname{Spec}(R)$ is homeomorphic to the one-point compactification of $\mathbf{N}$. In particular, $\operatorname{MaxSpec}(R)$ is Hausdorff but is not an $F$-space. By Theorem 4.5, $\partial_{e} \mathbf{P}(R)$ is homeomorphic to $\operatorname{Max} \operatorname{Spec}(R)$, hence $\partial_{e} \mathbf{P}(R)$ is not an $F$-space.

As we mentioned in the introduction to this section, many of our results-particularly Corollaries 4.3 and 4.6, Theorems 4.10 and 4.13, and Corollary 4.14-show that an $N^{*}$-complete regular ring $R$ behaves much like a ring of sections of a sheaf of simple self-injective rings. The obvious candidate for a topological space on which such a sheaf should live is $\operatorname{Max} \operatorname{Spec}(R)$. However, $\operatorname{MaxSpec}(R)$ is usually not Hausdorff, and even when it is Hausdorff it need not be disconnected, which would seem to be required for sheaf-theoretic proofs of results such as Corollary 4.14. The lack of Hausdorffness, at least, of $\operatorname{Max} \operatorname{Spec}(R)$ may be remedied by using the space $\partial_{e} \mathbf{P}(R)$ instead; the fact that we have to pay attention to how $\partial_{e} \mathbf{P}(R)$ sits inside $\mathbf{P}(R)$ is in some sense the price for overcoming the lack of compactness of $\partial_{e} \mathbf{P}(R)$. This feeling may be made more precise at the level of $K_{0}(R)$ : if $R$ were to resemble the sections of a sheaf based on $\partial_{e} \mathbf{P}(R)$, then $K_{0}(R)$ should resemble the sections of a sheaf of functions based on $\partial_{e} \mathbf{P}(R)$. When $\partial_{e} \mathbf{P}(R)$ is compact, this does happen: Combining Theorem 4.11 with [1, Proposition II.3.13] yields

$$
K_{0}(R) \cong\left\{q \in C\left(\partial_{e} \mathbf{P}(R), \mathbf{R}\right) \mid q(P) \in A_{P} \text { for all } P \in \partial_{e} \mathbf{P}(R)\right\} .
$$

On the other hand, when $\partial_{e} \mathbf{P}(R)$ is not compact, continuous real-valued functions on $\partial_{e} \mathbf{P}(R)$ do not correspond to elements of $K_{0}(R)$ unless they can be made to respect the affine relations present in $\mathbf{P}(R)$.

There is one situation in which $R$ is isomorphic to the ring of sections of a sheaf-like object, namely when $R$ is a continuous regular ring. This idea is developed by Handelman in [5] under the additional assumption that $R$ is $N^{*}$-complete; however, we now know that $N^{*}$-completeness holds for all continuous regular rings, by Theorem 1.8. Handelman's construction is based on the space $\partial_{e} \mathbf{P}(R)$, which in this case is a compact Hausdorff extremally disconnected space. (Of course, we could equally well use $\operatorname{Max} \operatorname{Spec}(R)$, in view of Theorem 4.5.) The stalk at a point $P \in \partial_{e} \mathbf{P}(R)$ is the simple self-injective ring $R / \operatorname{ker}(P)$. What prevents Handelman's object from being an actual sheaf is that the rank-metric topologies on the rings $R / \operatorname{ker}(P)$ must be respected, and these topologies are not discrete unless the rings $R / \operatorname{ker}(P)$ are artinian.

Another aspect of Handelman's development of this construction provides a general means for constructing $N^{*}$-complete regular rings. Namely, given any regular ring $R$, one can complete $R$ with respect to the $N^{*}$-metric. Since the ring operations on $R$ are uniformly continuous with respect to $N^{*}$ (as we observed in $\S \mathrm{I}$ ), the $N^{*}$-completion $\bar{R}$ is again a ring; moreover, $\bar{R}$ is actually a regular ring [6, Proposition 1.4]. In addition, the restriction map $\mathbf{P}(\bar{R}) \rightarrow \mathbf{P}(R)$ is an affine homeomorphism, as stated in [5, Proposition 15]. (The proof of this proposition is incomplete, but Handelman has informed me that he and Walter Burgess have developed a complete proof.) In particular, this result provides a convenient means 
for constructing examples. Combining it with [2, Theorem 17.23], we find that any metrizable Choquet simplex $S$ is affinely homeomorphic to $\mathbf{P}(R)$ for a suitable $N^{*}$-complete regular ring $R$. For instance, there exists an $N^{*}$-complete regular ring $R$ such that $\mathbf{P}(R)$ is affinely homeomorphic to the Choquet simplex of all probability measures on the unit interval $[0,1]$, whence $\partial_{e} \mathbf{P}(R)$ is homeomorphic to $[0,1]$.

A number of the results proved here for $N^{*}$-complete regular rings were first proved for $\boldsymbol{\aleph}_{0}$-continuous regular rings $[7,8]$, or, somewhat more generally, for unit-regular rings satisfying countable interpolation [4]. (All $\boldsymbol{\aleph}_{0}$-continuous regular rings, and their factor rings, satisfy countable interpolation by [4, Theorem II.12.3].) In the first case, our results are generalizations, since all $\boldsymbol{\aleph}_{0}$-continuous regular rings are $N^{*}$-complete (Theorem 1.8). However, in the second case our results are not strict generalizations except at the level of $K_{0}$ : Namely, a unit-regular ring $R$ satisfying countable interpolation need not be $N^{*}$-complete, but as long as $\operatorname{ker}(\mathbf{P}(R))=0$, then $K_{0}(R)$ is an archimedean norm-complete interpolation group [4, Theorems II.12.7 and I.6.6]. Thus the appropriate results for unit-regular rings with countable interpolation follow from the $K_{0}$ results in \$III, in the same manner as the derivations in $\S \mathrm{IV}$ for $N^{*}$-complete regular rings. The relationship between our results and these earlier results is as follows.

Theorem 4.2 corresponds to [4, Corollary II.13.6 and Theorem II.13.7], while Corollary 4.3 corresponds to [7, Corollary 3.2]. Theorems 4.5 and 4.9 correspond to [4, Proposition II.14.5 and Theorem II.14.6], while Corollary 4.6 and Theorem 4.10 correspond to [8, Theorem 2.3]. Theorems 4.11 and 4.13, along with Corollaries 4.12 and 4.14, correspond to [4, Theorems II.15.1, II.15.3, and II.15.4, and Corollary II.15.2].

NOTE ADDED IN PROOF. The $N^{*}$-completion results mentioned above are included in a paper by Burgess and Handelman, The $N^{*}$-metric completion of regular rings, submitted for publication.

\section{REFERENCES}

1. E. M. Alfsen, Compact convex sets and boundary integrals, Springer-Verlag, Berlin, 1971.

2. K. R. Goodearl, Von Neumann regular rings, Pitman, London, 1979.

3. K. R. Goodearl and D. E. Handelman, Metric completions of partially ordered abelian groups, Indiana Univ. Math. J. 29 (1980), 861-895.

4. K. R. Goodearl, D. E. Handelman and J. W. Lawrence, Affine representations of Grothendieck groups and applications to Rickart $C^{*}$-algebras and $\boldsymbol{\aleph}_{0}$-continuous regular rings, Mem. Amer. Math. Soc. No. 234 (1980).

5. D. Handelman, Representing rank complete continuous rings, Canad. J. Math. 28 (1976), 1320-1331.

6. Simple regular rings with a unique rank function, J. Algebra 42 (1976), 60-80.

7. Suppl. Studies, Vol. 4, 1979, pp. 171-196.

8. D. Handelman, D. Higgs and J. Lawrence, Directed abelian groups, countably continuous rings, and Rickart $C^{*}$-algebras, J. London Math. Soc. (2) 21 (1980), 193-202.

9. G. L. Seever, Measures on F-spaces, Trans. Amer. Math. Soc. 133 (1968), 267-280.

10. J. von Neumann, Continuous geometry, Princeton Univ. Press, Princeton, N. J., 1960.

Department of Mathematics, University of Utah, Salt Lake City, Utah 84112 